\title{
Asteroid Redirect Robotic Mission: Robotic Boulder Capture Option Overview
}

\author{
Daniel D. Mazanek ${ }^{1}$, Raymond G. Merrill ${ }^{2}$, Scott P. Belbin ${ }^{3}$, David M. Reeves ${ }^{4}$ and Kevin D. Earle ${ }^{5}$ \\ NASA Langley Research Center, Hampton, VA., 23681, USA \\ Bo J. Naasz ${ }^{6}$ \\ NASA Goddard Space Flight Center, Greenbelt, MD, 20771, USA \\ Paul A. Abell ${ }^{7}$ \\ NASA Johnson Space Center, Houston, TX 77058, USA
}

\begin{abstract}
The National Aeronautics and Space Administration (NASA) is currently studying an option for the Asteroid Redirect Robotic Mission (ARRM) that would capture a multi-ton boulder (typically 2-4 meters in size) from the surface of a large ( 100+ meter) Near-Earth Asteroid (NEA) and return it to cislunar space for subsequent human and robotic exploration. This alternative mission approach, designated the Robotic Boulder Capture Option (Option B), has been investigated to determine the mission feasibility and identify potential differences from the initial ARRM concept of capturing an entire small NEA (4-10 meters in size), which has been designated the Small Asteroid Capture Option (Option A). Compared to the initial ARRM concept, Option B allows for centimeter-level characterization over an entire large NEA, the certainty of target NEA composition type, the ability to select the boulder that is captured, numerous opportunities for mission enhancements to support science objectives, additional experience operating at a low-gravity planetary body including extended surface contact, and the ability to demonstrate future planetary defense strategies on a hazardous-size NEA. Option B can leverage precursor missions and existing Agency capabilities to help ensure mission success by targeting wellcharacterized asteroids and can accommodate uncertain programmatic schedules by tailoring the return mass.
\end{abstract}

\section{Introduction}

A key component of the National Aeronautics and Space Administration's (NASA's) Asteroid Initiative is the Asteroid Redirect Robotic Mission (ARRM). Following the 2011 Keck Institute for Space Studies (KISS) feasibility study ${ }^{1}$, NASA initiated a feasibility study of a mission to capture an entire small (approximately 4-10 meters in size) Near-Earth Asteroid (NEA) and redirect it to cislunar space where astronauts will explore it and return samples from it. The ARRM leverages a high specific impulse $\left(\mathrm{I}_{\mathrm{sp}}=2,000-3,000 \mathrm{~s}\right)$, high-power (40-50 kW) Solar Electric Propulsion (SEP) system in development by NASA's Space Technology Mission Directorate (STMD). ${ }^{2}$ An alternative ARRM option also being studied by NASA would capture a multi-ton boulder (typically 24 meters in size) from the surface of a larger ( $100+$ meter) NEA and return it to cislunar space for subsequent human and robotic exploration. ${ }^{3}$ Additionally, one or more planetary defense (PD) demonstrations would be performed while at the NEA. This mission approach, which is depicted in Fig. 1, has been designated the Robotic Boulder Capture Option (Option B) and a preliminary assessment has be performed to determine the mission

\footnotetext{
${ }^{1}$ Senior Space Systems Engineer, Space Mission Analysis Branch, E402/Mail Stop 462, Senior Member AIAA.

${ }^{2}$ Aerospace Engineer, Space Mission Analysis Branch (E402), Mail Stop 462, Senior Member AIAA.

${ }^{3}$ Senior Mechanical Engineer, Mechanical Systems Branch (D202), Mail Stop 432.

${ }^{4}$ Aerospace Engineer, Space Mission Analysis Branch (E402), Mail Stop 462, Senior Member AIAA.

${ }^{5}$ Aerospace Engineer, Space Mission Analysis Branch (E402), Mail Stop 462, Member AIAA.

${ }^{6}$ Project System Engineer, Satellite Servicing Capabilities Office (5990), Mail Stop 408, Senior Member AIAA.

${ }^{7}$ Lead Scientist for Planetary Small Bodies, Astromaterials Research and Exploration Science Directorate (KR111), Mail Stop KR.
} 


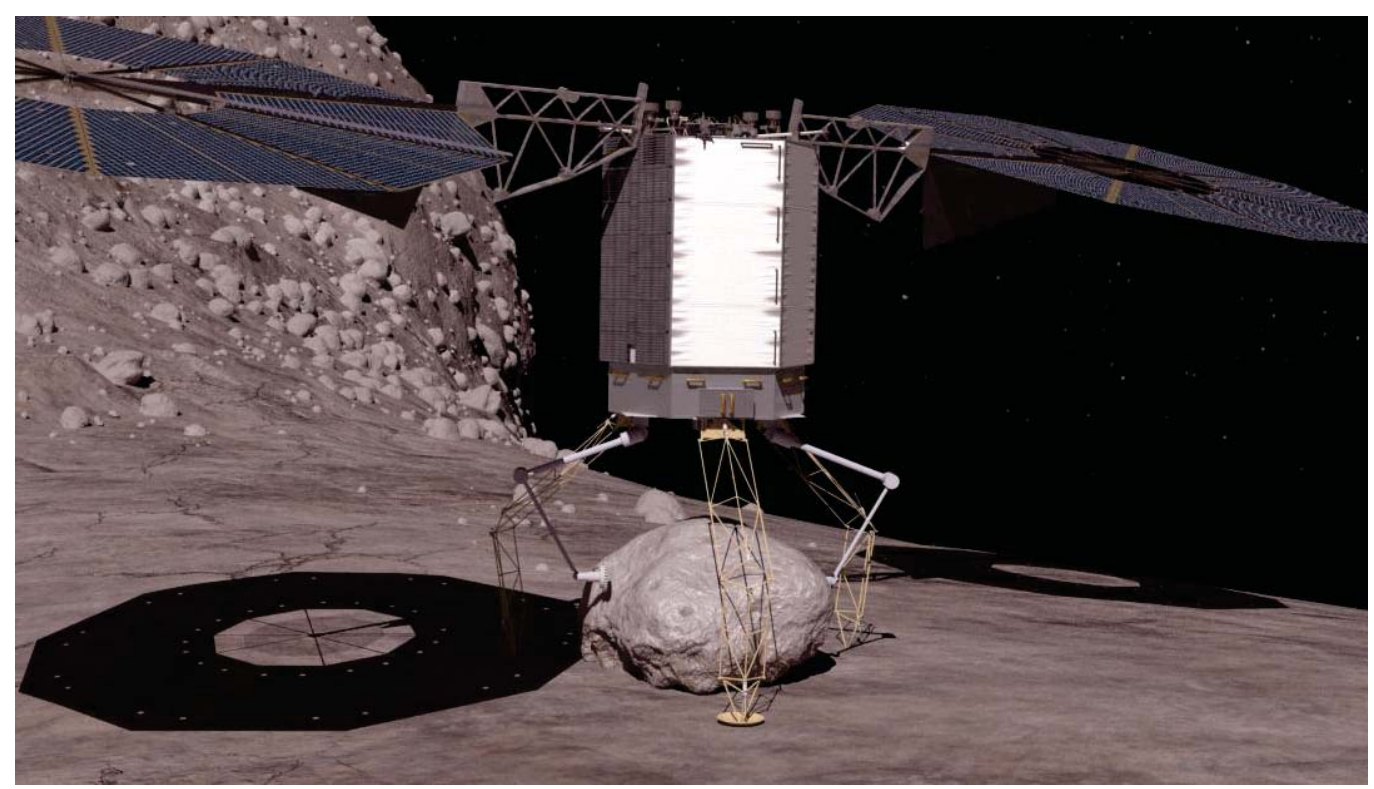

Figure 1: ARRM Robotic Boulder Capture Option (Option B) (Image Credit: NASA/AMA, Inc.).

feasibility and identify potential differences from the initial ARRM concept, which has been designated the Small Asteroid Capture Option (Option A). This mission approach provides NASA the option to trade potential return mass for centimeter-level characterization over an entire large NEA, the ability to select the NEA's composition type (i.e., a water/volatile-rich carbonaceous target), the ability to select a well-characterized, science community selected sample with geological context, additional experience operating at a low-gravity planetary body including extended surface contact critical to future human and robotic missions, and the ability to demonstrate future deflection strategies on a NEA of a relevant size which will inform strategies to defend our home planet.

The ARRM, with a planned launch date in 2019, is one of three components of the Asteroid Redirect Mission (ARM). The second component is the Asteroid Redirect Crewed Mission (ARCM) in which astronauts aboard NASA's Orion spacecraft launched on the Space Launch System (SLS) will rendezvous with the returned asteroid or boulder in a Lunar Distant Retrograde Orbit (LDRO), conduct spacewalks to collect samples, and return the samples to the Earth for analysis. The ARCM is planned for the mid 2020's. The third ARM component, which is currently being conducted, is an observation campaign to identify and characterize candidate NEAs using ground-based facilities including optical telescopes, radar facilities, and NASA's Infrared Telescope Facility (IRTF), and spacebased assets, such as NASA's Spitzer Infrared Space Telescope and Near-Earth Object Wide-field Infrared Survey Explorer (NEOWISE) Space Telescope. The same assets are being used to help catalog the general population of NEAs and identify those that are a threat to impact the Earth.

The capabilities, systems, and operational experience developed and implemented by the ARRM, ARCM, and subsequent missions to the returned asteroidal material will advance NASA's goal of sending humans beyond lowEarth orbit (LEO) and on the path to future missions to Mars. The extensibility of these missions is discussed in further detail in Section X.

\section{Stakeholder Benefits}

The ARRM Option B approach addresses the needs of a broad set of stakeholders, including human exploration, planetary defense, science, and commercial and international partnerships. Additionally, the mission approach leverages precursor missions and existing Agency capabilities to enable mission success. This mission option supports future human exploration by addressing multiple Mars-forward technology and operations gaps including operations around and on the Martian moons, Phobos and Deimos. The approach also provides a well-characterized, accessible, multi-ton boulder for astronauts to explore and return samples from, while also being robust to programmatic uncertainties.

In addition to providing a near-term destination for crews to explore, Option B advances various aspects of robotic systems and autonomous operations critical to human exploration and robotic missions. The SEP system,

2

American Institute of Aeronautics and Astronautics 
which is used by both options, will enable a wide range of human missions in cislunar space, to NEAs, and to the Mars system, as well as robotic exploration of the solar system. The ARRM systems, subsystems, and technologies can be evolved to facilitate more demanding missions through both modularity and scalability.

In the area of planetary defense, Option B permits the demonstration of one or more orbital deflection techniques on a relevant, hazardous-size NEA. Although Option A permits the demonstration of deflection techniques on a very small NEA that can be verified more rapidly, these small NEAs are not a significant threat to the Earth's inhabitants as atmospheric entry will typically completely ablate NEA's less than 10 meters. Additionally, Option B provides the opportunity to test a kinetic impact technique, utilizing the Asteroid Redirect Vehicle (ARV) as an observer spacecraft, and provides extended surface interaction with a hazardous-size NEA, both of which are likely critical to future efforts to deflect or disrupt an Earth-impacting asteroid or comet.

Science community interest is focused on primitive, water/volatile-rich carbonaceous targets that could provide valuable information on the formation of our solar system and the origins of life on Earth. Option B allows the return of a well-characterized, science community selected sample with geological context from a NEA of a known compositional type (i.e., a large carbonaceous boulder and one or more parent body surface samples). The mission option also provides numerous opportunities for mission enhancements to support science objectives, including: robotic arm sample collection (e.g., drilling, coring, surface sample collection, and caching); the ability to host and deploy free-flyers or surface rovers/hoppers; and additional scientific instrumentation for in-situ or remote observation of a large NEA. Additionally, the advanced SEP technologies, instrumentation, and low-gravity body autonomous operations will help enable more capable future robotic missions.

The mission option offers important benefits to commercial and international partners, by providing critical operational experience interacting with a large, low-gravity body and collecting multiple tons of asteroidal material, access to a large surface area of potentially volatile/water-rich carbonaceous material, and the opportunity for hosted payloads including In-Situ Resource Utilization (ISRU) experiments and demonstrations. The return of many tons of carbonaceous material to cislunar space would significantly accelerate the efforts of the fledgling asteroid mining industry, which in turn will aid humanity's expansion into the solar system by tapping the vast resources available from asteroids.

\section{Flight System Overview}

The ARRM Option B flight system shares many similarities with the initial ARRM approach of returning an entire small NEA. Only minimal modifications are required for Mission Module and SEP Module on the Asteroid Redirect Vehicle (ARV), and the required sensor suite is also very similar. Conversely, the capture system and the proximity operations are significantly different. Due to the coherent nature of the boulder that will be collected, encapsulation of the asteroidal material is not required and several autonomous manipulator-based capture system options have been investigated. The lack of encapsulation also facilitates exploration and sample collection after the boulder is returned to the Earth-Moon system. The current capture system design has focused on accommodating a free-standing boulder that is one to four meters in size (up to a mass of $\sim 70 \mathrm{t}$ ), but the design can be scaled to accommodate up to at least 10 meter boulders. This allows the capture system to be tailored to handle the maximum mass returnable from the selected asteroid. Currently, there are no target NEAs that provide the higher mass-return opportunities needed for these larger sizes in the timeframe being considered for the ARRM.

The Option B ARV is designed in a modular fashion to both enable reduction in the schedule required for integration and test (see Section IX) and to allow for module design re-use in the future. The spacecraft flight system consists of the following three modules: 1.) SEP Module; 2.) Mission Module; and 3.) Capture Module. Some of the Option B module characteristics and how they differ from Option A are identified in Fig. 2. Depicted is a capture system option that utilizes a space frame structure for both the capture and contact arms. Section VI provides details on additional Capture Module options investigated.

The ARRM Option B approach utilizes the same SEP Module concept that as Option A. The SEP Module includes a pair of solar arrays that generate $51 \mathrm{kWe}$ at beginning of life at $1 \mathrm{AU}$. Two possible solar array configurations are shown in Fig. 3. The upper configuration is the Roll-Out Solar Array (ROSA) being developed by Deployable Space Systems (DSS). Each wing is approximately 4.5 meters wide and 14 meters long. The lower configuration is ATK's MegaFlex solar array and consists of two circular arrays that are each approximately $9.6 \mathrm{~m}$ (32 ft) in diameter. The SEP Module Electric Propulsion System (EPS) is designed for $40 \mathrm{kWe}$ input power that uses a 300 V Power Processing Unit (PPU) architecture and 4 or 6 magnetically shielded hall thrusters that operate with an $\mathrm{I}_{\mathrm{sp}}$ between 1,800 and 3,000 s and are 50-55\% efficient depending on input power. Body-mounted, louvered heat pipe radiators provide thermal control. The SEP Module also includes the xenon feed system for each thruster and 12 xenon tanks capable of holding a total of 11.1 t of xenon (including $11 \%$ margin). The SEP Module also includes 


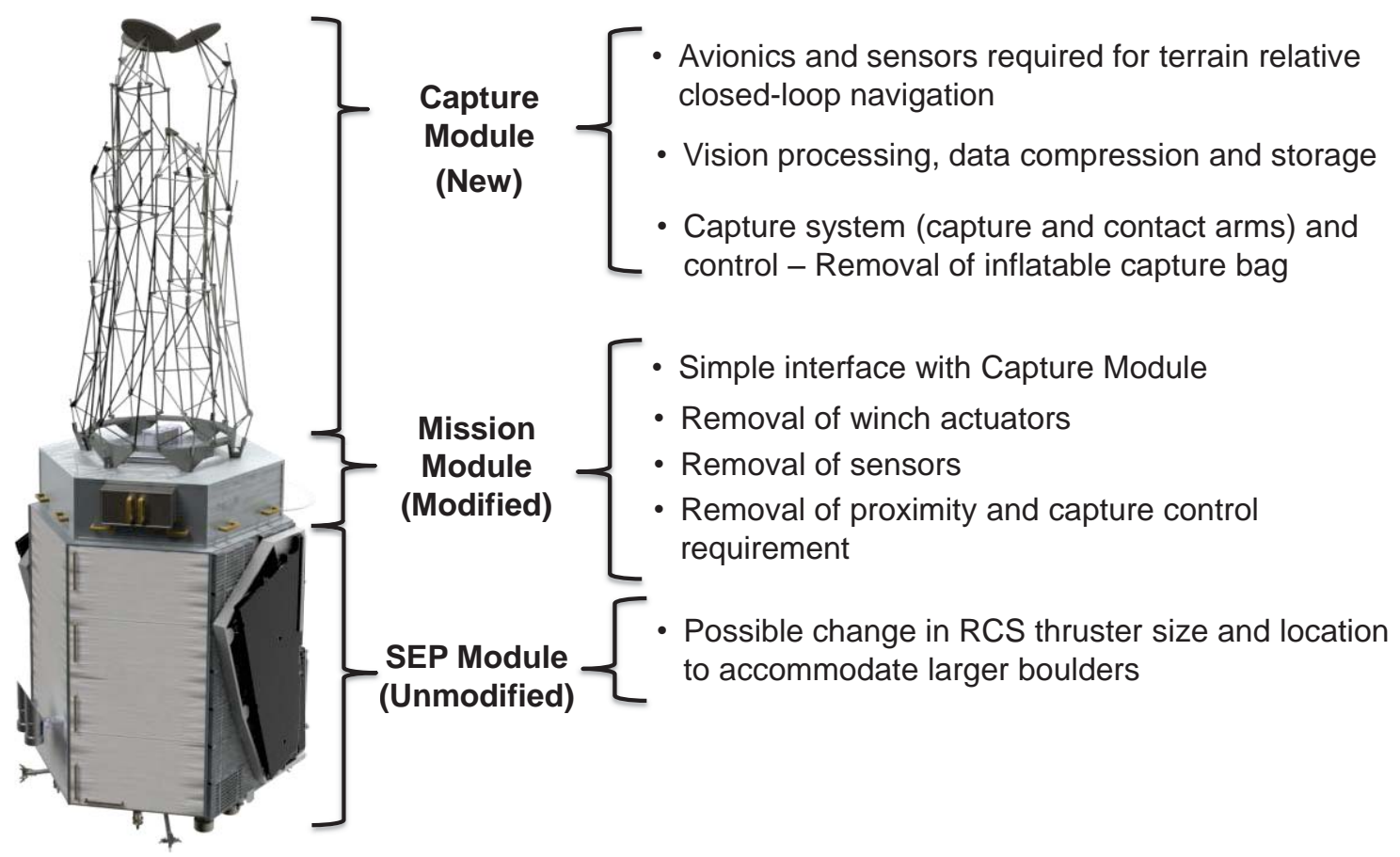

Figure 2. Asteroid Redirect Vehicle (Stowed Configuration)

Comparison with Option A (Image Credit: NASA/AMA, Inc.).
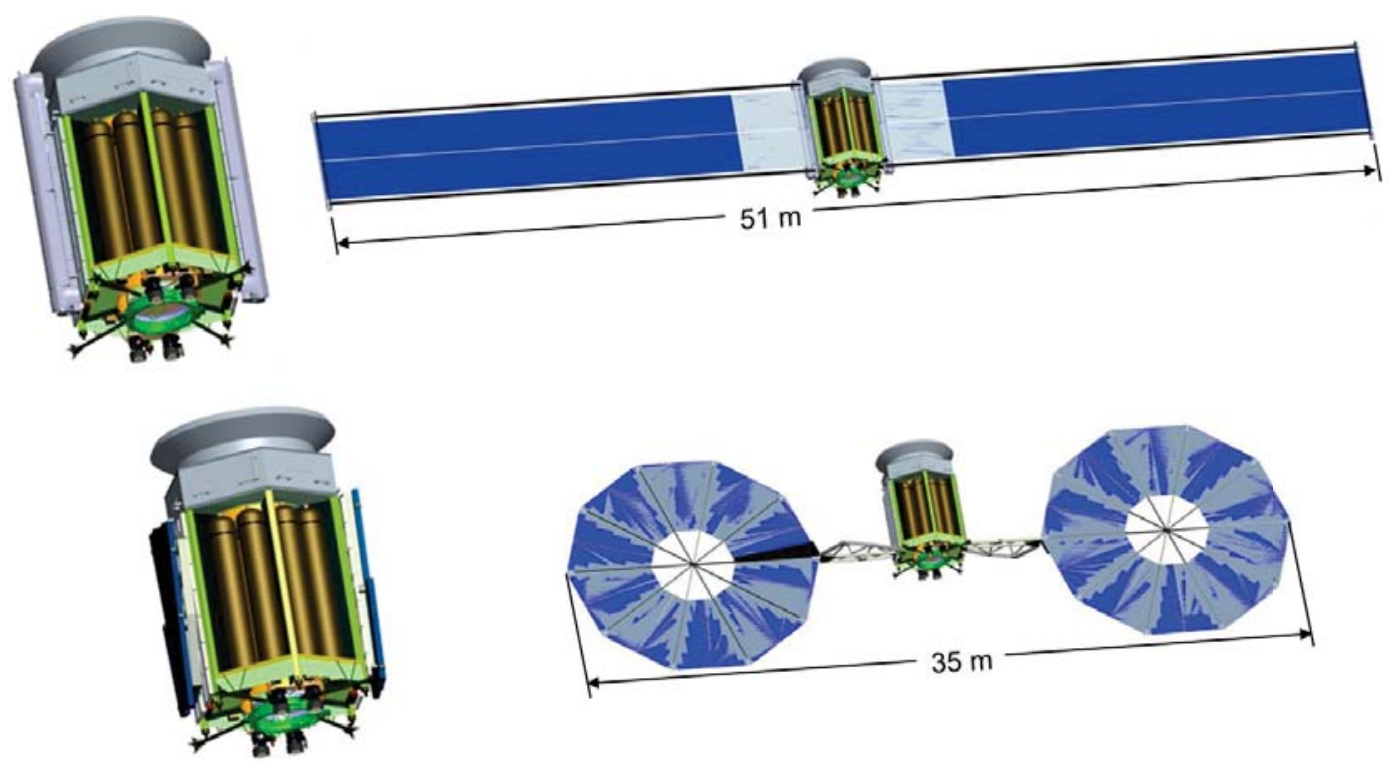

Figure 3. Possible ARV Solar Array Configurations (Image Credit: NASA/JPL).

a Reaction Control System (RCS) with a 400 kg mono-propellant tank and 4 thruster pods which are currently preintegrated. For very large boulders (greater than $~ 70 \mathrm{t}$ ) larger RCS thrusters may be required to facilitate ascent from the surface of the selected NEA. At the aft end of the SEP Module is docking interface that allows the Orion MultiPurpose Crew Vehicle to attach to the ARV during the ARCM.

The SEP Module is controlled by the Mission Module, which provides the main command and data handling capability for the overall vehicle. The Mission Module is responsible for ground communications and ranging, and control of the vehicle actuators (SEP and chemical thrusters in the SEP Module and momentum wheels in the Mission Module). During the asteroid proximity operations phase, the Mission Module receives onboard navigation 
information from the Capture Module, executing an autonomous sequence to descend to the surface, collect the boulder, and return to a safe trajectory. To support the crewed phase, the Mission Module hosts a tool stowage box and provides crew translation aids between the Orion spacecraft and Capture Module. Together, the SEP Module and Mission Module form a functional spacecraft bus that can be utilized for a variety of future missions.

The Capture Module provides all functions necessary to perform the onboard asteroid-relative and boulderrelative navigation, asteroid surface interaction, boulder capture, boulder restraint during the ascent and return flight. It also provides enhanced hardware to support crew extra-vehicular activity (EVA), including robotic preparation of the work site prior to crew arrival and between EVAs, and potentially robotic caching of boulder surface and subsurface samples for crew evaluation, collection, and return to Earth. Additional details on the Capture Module are provided in Section VI.

\section{Target Selection and Mission Design}

Characterization of a candidate NEA is a critical aspect of the asteroid being selected as the target for the ARRM. High-quality remote characterization utilizing ground-based and space-based assets can be extremely beneficial, allowing Option B targets to be selected based on sufficient knowledge about the NEA (size, spin state, composition type, presence of boulders, etc.). However, remote characterization has limitations and in-situ reconnaissance from a precursor mission can provide the detailed information that can help ensure mission success. Of the four asteroids larger than $100 \mathrm{~m}$ that have been or may be visited by robotic spacecraft, three could be potential targets for the ARRM mission.

While numerous Near-Earth Asteroids larger than $100 \mathrm{~m}$ have been discovered and remotely characterized, only Itokawa (visited by the Japanese Hayabusa spacecraft in 2005) and Eros (visited by NASA's Near Earth Asteroid Rendezvous (NEAR)-Shoemaker spacecraft in 2000-2001) are currently well-characterized. Itokawa, which is a stony (S-type) NEA, provides an attractive destination for Option B by providing hundreds of candidate boulders of various dimensions (see Section V). Itokawa's orbit and the desire to return the boulder to cislunar space by 2025 limit the return mass to approximately 5 metric tons. Extending the return date by as little as one year can increase the return mass by a factor of approximately four. Eros is not a viable candidate due to the lack of opportunities to return mass in the timeframe being considered for the ARRM.

Based on Hayabusa's characterization of Itokawa, it has been utilized to develop a detailed mission design to assess options and risks associated with proximity operations and to understand spacecraft design requirements. However, it is not the only candidate NEA for Option B with the possibility of extensive precursor reconnaissance. NASA's Origins-Spectral Interpretation-Resource Identification-Security-Regolith Explorer (OSIRIS-REx) spacecraft will visit Bennu in 2018 and the Japan Aerospace Exploration Agency's (JAXA's) Hayabusa 2 spacecraft will rendezvous with $1999 \mathrm{JU}_{3}$ in 2018. Both will provide detailed characterization of their physical properties. One additional candidate, $2008 \mathrm{EV}_{5}$, has been highly characterized by ground-based radar (signal-to-noise ratio > 5000) and in the infrared wavelengths with the Wide-field Infrared Survey Explorer (WISE) spacecraft. Based on groundbased reconnaissance, all three of these NEAs are carbonaceous chondrite targets (C-types) and are believed to be water/volatile-rich and may contain significant amounts of organic materials.

In addition to these four potential Option B targets, there exists the possibility that additional NEAs could be identified and sufficiently characterized using radar observations prior to mission launch. A scan using a ranking parameter and a thrust-limited, multi-revolution Lambert's solver identified numerous asteroids with ballistic trajectories with a low velocity change $(\Delta \mathrm{V})$ needed for significant mass return. Both the Mission Analysis LowThrust Optimization (MALTO) tool and the Evolutionary Mission Trajectory Generator (EMTG) software tool were used to perform global searches for low-thrust, gravity-assist trajectories to potential targets. ${ }^{4}$ Trajectories to over 60 Option B target NEAs with upcoming radar observations were found, but opportunities for observations with high signal-to-noise ratios are limited. The existence of a well-characterized target (Itokawa), three high-value carbonaceous chondrite targets (two with planned precursor missions), and the possibility of additional targets being identified and remotely characterized provides a robust set of target NEAs for Option B.

The ARRM trajectory design is complex for a variety of reasons and an overview of the Option B mission design and comparison with Option A is provided in Fig. 4. While the ARV's SEP system offers the propellant efficiency required to return a massive asteroid boulder to an orbit in cislunar space, it is only capable of relatively low-thrust $(<2 \mathrm{~N})$. Thus, long periods of thrust are required to achieve the necessary $\Delta \mathrm{V}$ for a mission, and low-thrust trajectory optimization is necessary to identify feasible trajectories composed of long-thrust arcs. Additionally, for any candidate NEA the return mass is subject to the interdependence between mission duration, launch date, launch vehicle capacity, and spacecraft performance. The return mass is maximized in the interplanetary trajectory and used to inform proximity operations analysts regarding the largest boulder mass that can be captured and redirected. The 
programmatic desire to return the ARV to the Earth-Moon system for crew availability by the end of 2024 places a strict upper bound on the mission time of flight and eliminates trajectory options that might allow for more optimal phasing with a later return date, which would allow a higher return mass. In addition, extended ARV operations at the NEA (e.g., one year or more) for characterization and planetary defense operations decreases trajectory options due to the reduced time available to thrust. Later return dates, later launch dates, and reduction in stay time have also been assessed to illustrate the effect of programmatic decisions and uncertainties on the maximum boulder return mass.

Design space complexity is increased by the possible inclusion of a planetary gravity assist to reduce $\Delta \mathrm{V}$ requirements. NEA boulder retrieval missions can benefit from energy- and plane-altering flybys of Earth, Mars, or Venus, warranting a thorough search for gravity assists on both the outbound and inbound legs. This design space grows significantly when considering target asteroids in addition to those that have had or will have precursors before launch.

For ARRM Option B missions launched on a Falcon Heavy or Delta IV Heavy, a pair of lunar gravity assists increases the departure characteristic energy (C3) from $-2 \mathrm{~km}^{2} / \mathrm{s}^{2}$ to $2 \mathrm{~km}^{2} / \mathrm{s}^{2} ., 6$ Interplanetary trajectory modeling was simplified by assuming a maximum Earth departure mass based on the launch vehicle capability to a C3 of -2 $\mathrm{km}^{2} / \mathrm{s}^{2}$ and an upper bound on the departure C3 of $2 \mathrm{~km}^{2} / \mathrm{s}^{2}$ with a declination between $+/-30$ degrees. For the Falcon Heavy, the maximum spacecraft mass was approximately $13.2 \mathrm{t}$. These values include mass decrements for the propellant assumed to be necessary for any maneuvers to compensate for launch vehicle insertion errors and perform phasing before lunar flybys. The ARV dry mass plus the reaction control system (RCS) propellant limits the available xenon for low-thrust propulsion. The ARV dry mass plus RCS propellant was $5.2 \mathrm{t}$ for the 2,000 s $\mathrm{I}_{\mathrm{sp}}$ EPS configuration and $5.0 \mathrm{t}$ for the $3,000 \mathrm{~s} \mathrm{I}_{\mathrm{sp}}$ EPS configuration.

Once at the NEA, the ARV will conduct proximity operations to characterize the asteroid, collect the boulder, and conduct planetary defense demonstration(s). The time required for characterization is dependent on whether a precursor mission has already been conducted, with less time required for a previously characterized asteroid such as Itokawa. The time allocation for boulder collection includes a budget for five collection attempts at up to three different locations on the NEA. Time is also budgeted for PD demonstration operations and measuring the change in the NEA's orbit. Specifically, a gravity tractor demonstration using the captured boulder to augment the mass of the ARV has been assumed as the primary PD demonstration (see Sections VII and VIII). This planetary defense demonstration is dependent on the mass of the NEA, the mass of the collected boulder, and the ARV thrust

\section{\# Similar to Option A}

\section{\# Different from Option A}

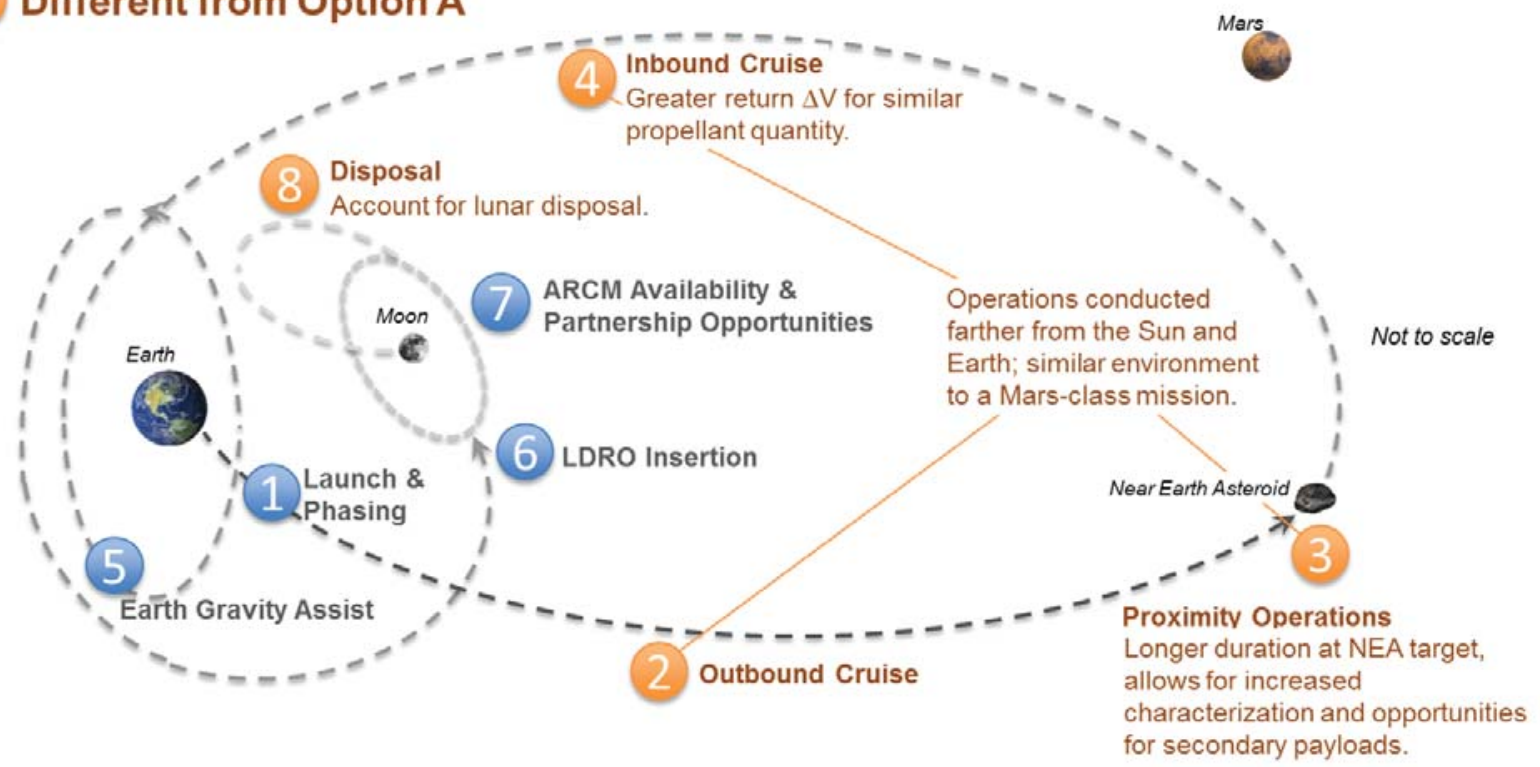

Figure 4: Option B Mission Design Overview and Comparison with Option A. 
capability. It needs to be conducted long enough to perturb the orbital state of the NEA outside of the navigation uncertainty bounds of the nominal, unaltered orbit, along with enough elapsed time for the perturbation to reach a measurable level and for the Earth-ARV orbital geometry to permit this measurement. For Itokawa, the minimum stay time at the asteroid was determined to be 400 days for a conservative design. This stay time is also used as an estimate for the other asteroids examined with the understanding that the stay time would change after a detailed proximity operations design for each particular target NEA is performed. Additionally, a reduced stay time of 120 days was also examined as the likely lower limit for boulder collection and a shortened planetary defense demonstration.

Trajectories were generated for Itokawa, Bennu, $1999 \mathrm{JU}_{3}$, and $2008 \mathrm{EV}_{5}$ for Delta IV Heavy, Falcon Heavy, and SLS launch vehicles. The maximum boulder return masses in metric tons for three different Earth system departure periods (2019, 2020, and 2021) and six different Earth system arrival periods (2024-2029) with a Falcon Heavy launch are shown in Table 1. The one year launch periods are defined assuming a launch window that opens no earlier than June $1^{\text {st }}$, resulting in the ARV leaving the Earth-Moon no earlier than September $23^{\text {rd }}$ when utilizing lunar gravity assist. The arrival period in the table is defined as one year starting on January $1^{\text {st }}$ of the listed year. The highest return mass identified is reported in Table 1, where both 2,000 s and 3,000 s $\mathrm{I}_{\mathrm{sp}}$ electric propulsion systems were investigated in conjunction with both direct and gravity assist options.

Table 1. Maximum Boulder Return Mass (metric tons) for Candidate NEAs with a Falcon Heavy Launch Vehicle.

\begin{tabular}{l|lccccc|} 
Itokawa & \multicolumn{7}{c}{ Crew Availability in LDRO } \\
& 2024 & 2025 & 2026 & 2027 & 2028 & 2029 \\
2019 & 0.2 & 5 & 19 & 20 & 20 & \\
2020 & & & 10 & 14 & 12 & \\
2021 & & & & 0 & 8 & 34 \\
\hline
\end{tabular}

\begin{tabular}{rlllllll|}
$1999 \mathrm{JU}_{3}$ & \multicolumn{7}{c}{ Crew Availability in LDRO } \\
& 2024 & 2025 & 2026 & 2027 & 2028 & 2029 \\
\cline { 3 - 9 } 2019 & 1 & 13 & 29 & 45 & & \\
2020 & & & 23 & 43 & 47 & \\
2021 & & & & 11 & 14 & 14 \\
\hline
\end{tabular}

\begin{tabular}{rlcccccc|} 
Bennu & \multicolumn{7}{c}{ Crew Availability in LDRO } \\
\cline { 2 - 8 } 2019 & 2024 & 2025 & 2026 & 2027 & 2028 & 2029 \\
\cline { 2 - 8 } & 6 & 12 & 23 & 27 & 28 & \\
2021 & & & 12 & 16 & 16 & \\
\hline & & & & 8 & 12 & 17 \\
\hline
\end{tabular}

\begin{tabular}{|c|c|c|c|c|c|c|}
\hline \multirow{2}{*}{2008 EV } & \multicolumn{6}{|c|}{ Crew Availability in LDRO } \\
\hline & 2024 & 2025 & 2026 & 2027 & 2028 & 2029 \\
\hline 2019 & 11 & 32 & 40 & 51 & & \\
\hline 2020 & & & 43 & 44 & 57 & \\
\hline 2021 & & & & 27 & 41 & 43 \\
\hline
\end{tabular}

An Earth Gravity Assist (EGA) on the return leg frequently increased the maximum return mass for missions with total duration greater than 5 years. EGAs are especially beneficial for asteroids with relatively high inclinations, where the Earth flyby can effectively enable a propellant-free plane change. In the case of $2008 \mathrm{EV}_{5}$, with an inclination of 7.4 degrees from the ecliptic, an EGA improved the maximum boulder return mass by $80 \%$ for the dates shown. Fig. 5 shows the boulder mass and size for a June 2019 launch and crew availability in cislunar space between February-May of 2025 for the three launch vehicles and four candidate asteroids assessed. The size

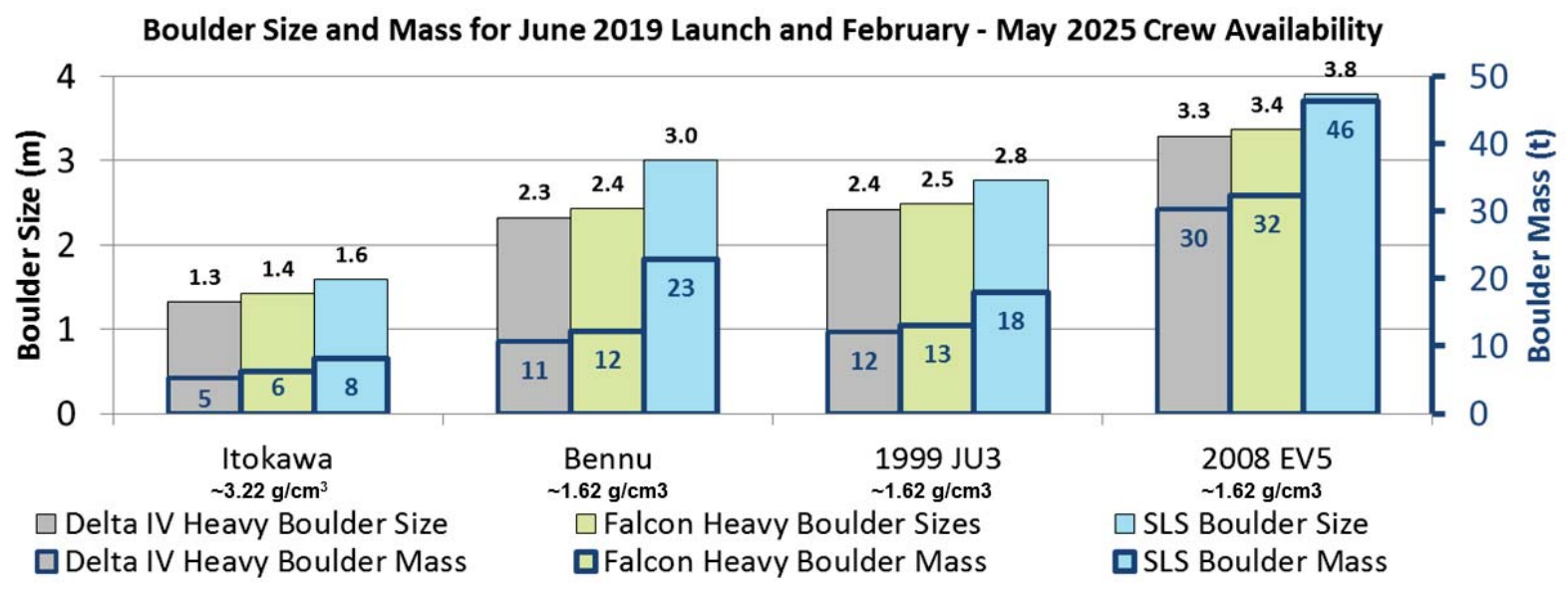

Figure 5. Comparison of Boulder Size and Mass Return for Candidate Launch Vehicles and NEAs. 
of boulder is significantly larger for the three carbonaceous targets due to both the higher return mass and the lower density assumed for these targets $\left(1.62 \mathrm{~g} / \mathrm{cm}^{3}\right.$ vs. $\left.3.22 \mathrm{~g} / \mathrm{cm}^{3}\right)$. An example of the interplanetary trajectory for a Bennu boulder return mission launching in 2019 and returning to the Earth-Moon system in 2024 is plotted in Fig. 6. This trajectory assumes a 120 day stay time, the use of the SLS and an ARV utilizing an EPS with an $\mathrm{I}_{\mathrm{sp}}$ of 2,000 s. The red lines represent the three-dimensional EPS thrust direction at different point in the trajectory represented in a two-dimensional plot.
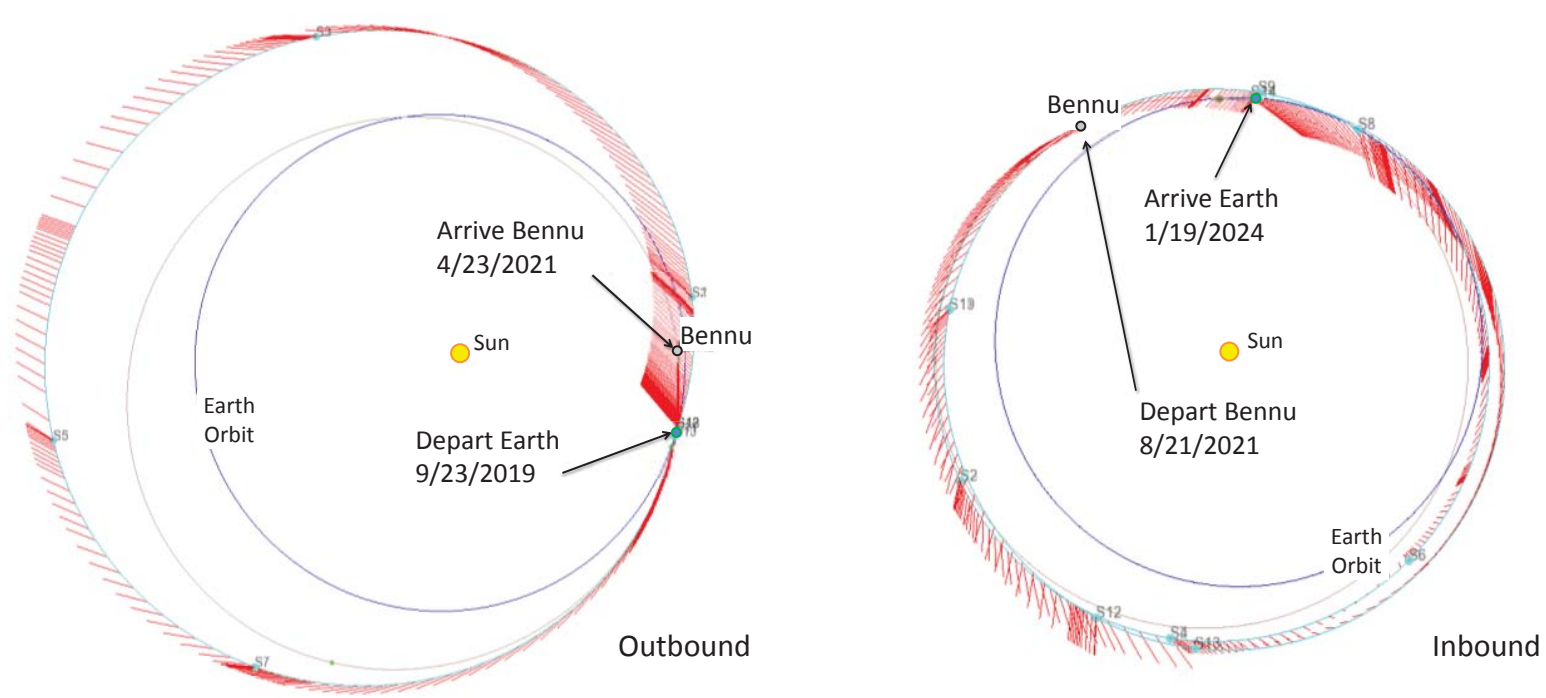

Figure 6. Example Interplanetary Trajectory for Bennu with a 120 Day Stay.

\section{NEA and Boulder Characteristics}

NEAs are generated through various dynamical and collisional interactions within the asteroid main belt, and range from objects as small as a few meters to several tens of kilometers in diameter. The NEA population of objects has a wide range of physical properties and characteristics as understood from the information obtained from the analysis of meteorites, ground- and space-based observations of small bodies, and robotic spacecraft missions. Many different types of compositions are thought to exist among the NEA population, and it is estimated that there may be in excess of at least 100 different parent bodies from which these objects originated. ${ }^{7}$ In addition to the compositional variety, NEAs also appear to have a wide range of shapes, internal structures, and configurations. ${ }^{8}$ These objects can be intact polygonal monoliths, heavily-fractured but coherent prolate bodies, or highly porous rubble piles. Some of these objects even form as binary or ternary systems. NEAs also have a wide range of possible spin rates and states. The NEA targets identified for Option B possess rotational periods of approximately 4-12 hours, compared to possible rotational periods as short as two minutes assumed for small NEAs targeted by Option A.

The best observations of NEAs and their physical properties have been made by robotic spacecraft flyby or rendezvous missions (e.g., NASA's NEAR-Shoemaker, Japan's Hayabusa, and China's Chang'e 2). These missions have allowed a much better understanding of the surfaces and internal characteristics of NEAs. One of the most successful NEA missions to date has been the Hayabusa mission to Itokawa. Hayabusa determined that Itokawa has a porosity of $\sim 40 \%$ and is a rubble-pile asteroid. ${ }^{9}$ Images from the Hayabusa mission, as shown in Fig. 7, indicate that $\sim 20 \%$ of the asteroid's surface is smooth terrain. These terrains are wide, flat areas with relatively few hazards, but contain many boulders. One of the largest areas is the Muses Sea region, which is approximately $60 \mathrm{~m}$ across (smooth region visible in the lower image of Fig. 7). These areas are dominated by pebble size particles and stereo analysis suggests that some boulders in these regions are not embedded. ${ }^{10}$ Boulder counts from the Hayabusa images have identified thousands of boulders on Itokawa's surface as shown in Fig. 8, which plots the observed number $(\mathrm{N})$ of boulders (also referred to as "blocks") per square kilometer greater than a particular diameter (D) for the lowlands and highlands, along with the global distribution of boulders greater than six meters. ${ }^{11}$ The total surface of Itokawa is approximately $0.4 \mathrm{~km}^{2}$, so Itokawa possesses hundreds of candidate boulders, many of which are located in very accessible regions. The presence of boulders on the surface of NEAs and the mechanism by which they are produced 
is thought to be relatively common based on the data from the Hayabusa mission, remote sensing observations, and dynamical modelling. To date, boulders have been seen on every NEA visited by spacecraft.

Recent radar observations of Bennu suggest the presence of boulders on its surface which will be confirmed by OSRIS-REx well before the launch of the ARRM..$^{12}$ No radar observations have yet been made of 1999 JU3, but Hayabusa 2 will be able to observe and characterize any boulders on its surface, also before launch of the ARRM. $2008 \mathrm{EV}_{5}$ was considered as the prime target of the European Space Agency's (ESA's) Marco Polo-R sample return mission and has been well-characterized via ground-based assets. The planetary radar observations of this target suggest that it also has numerous boulders on its surface, which would make it attractive target for Option $\mathrm{B} .{ }^{13}$ The manner in which these boulders are generated and their evolutionary history implies that they are relatively coherent objects. The composition of the NEA also plays a factor into the inherent strength of the boulders on its surface. Itokawa has been determined to have an LL ordinary chondrite composition (LL stands for Low iron and Low metal) based on samples returned to Earth in 2010 via the Hayabusa mission. ${ }^{14}$ Such objects have compressive strengths on the order of a few tens to hundreds of megapascals (MPa). Other NEAs under consideration as potential targets likely have compositions with lower strength, but even carbonaceous chondrite targets have compressive strengths from 0.3 to $50 \mathrm{MPa}$. Hence these boulders are unlikely to fragment during the collection process given the magnitude of the forces applied by the capture system mechanisms.

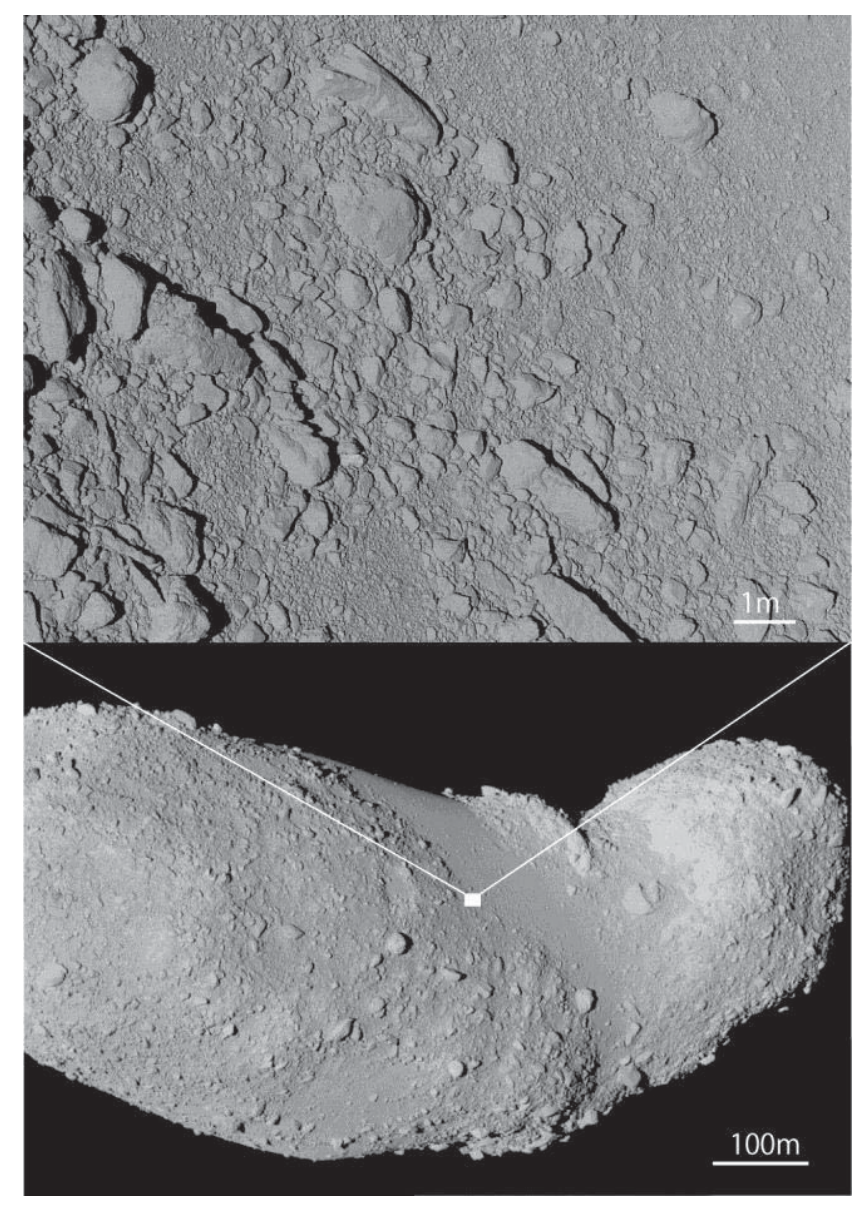

Figure 7: Itokawa and Boulders (Image Credit: JAXA). 


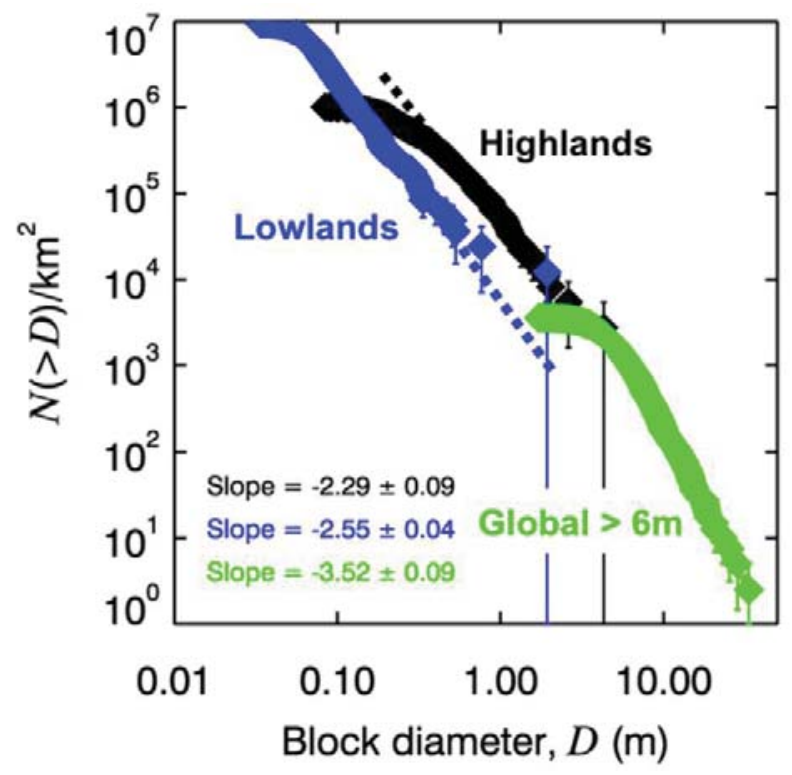

Figure 8. Observed Boulder Population on Itokawa (Credit: Ref. 11).

\section{Capture Module Design and Options}

The Capture Module provides all of the functions necessary to perform the following key functions: 1) asteroid and boulder characterization; 2) onboard asteroid- and boulder-relative navigation; 3) asteroid surface interaction; 4) boulder capture; 5) boulder restraint during the return flight; and 6) enhanced support of crew extra-vehicular activity (EVA), including robotic preparation of the work site prior to crew arrival and between EVAs, and potential robotic collection and caching of boulder surface and sub-surface samples for crew evaluation, collection, and return to Earth. Further details on the capture system design options investigated for Option B can be found in Ref. 15.

As shown in Fig. 9, several boulder collection options have been considered that focus of surface contact operations and actuator type. The two surface interaction options investigated are sustained contact (or "landing") and hovering. In the contact option, the Capture Module provides relative navigation information to the Mission Module, which commands SEP Module chemical thrusters to descend to the boulder site with the contact arms (or "legs") straddling the boulder, and thrusters maintaining continuous contact with the asteroid surface. The capture arm(s) are then used to collect the boulder.

In the hover option, the Capture Module provides relative navigation information to the Mission Module, which commands SEP Module chemical thrusters to hover over the boulder, while the capture arm(s) secure the boulder. This option removes the need for landing legs, but also stresses the vehicle Guidance, Navigation and Control (GN\&C) subsystem more, and increases the potential for regolith/dust ejection from the asteroid surface due to the increased requirement for thrusting towards the surface.

The actuator type options shown in Fig. 9 are Spaceframes, robotic arms, and a hybrid system including both. In the Spaceframe only configuration, 3- or 4-Degree-of-Freedom (DOF) trusses articulated by linear actuators are arranged in two configurations (legs and capture arms) of three appendages each to perform the surface contact and boulder capture functions. In the arm-only configuration, identical 7-DOF arms are used. The hybrid configuration uses both systems, with three Spaceframe surface contact arms and two robotic capture arms.

The hybrid version of the Capture Module includes the following subsystems to perform the functions described above: Relative Navigation Subsystem; Robot Subsystem; Contact and Restraint System (CRS); Avionics Subsystem; and Mechanical Subsystem. Current mission refinement efforts are focused on the hybrid option, which is discussed in detail below.

The Relative Navigation Subsystem (RNS) consists of a highly capable sensor suite, and data processing algorithms (hosted in the Avionics Subsystem's SpaceCube computers) to perform both the machine vision and filtering required for onboard Terrain-Relative Navigation (TRN). The fully redundant sensor suite consists of three high-definition cameras with differing field of views and a 3D LIDAR. The Narrow Field of View (NFOV) and Medium Field of View (MFOV) cameras are located on a side mounted two-axis gimbal to provide high-resolution mapping during asteroid flybys and extended horizon views for onboard navigation during the descent and planetary 


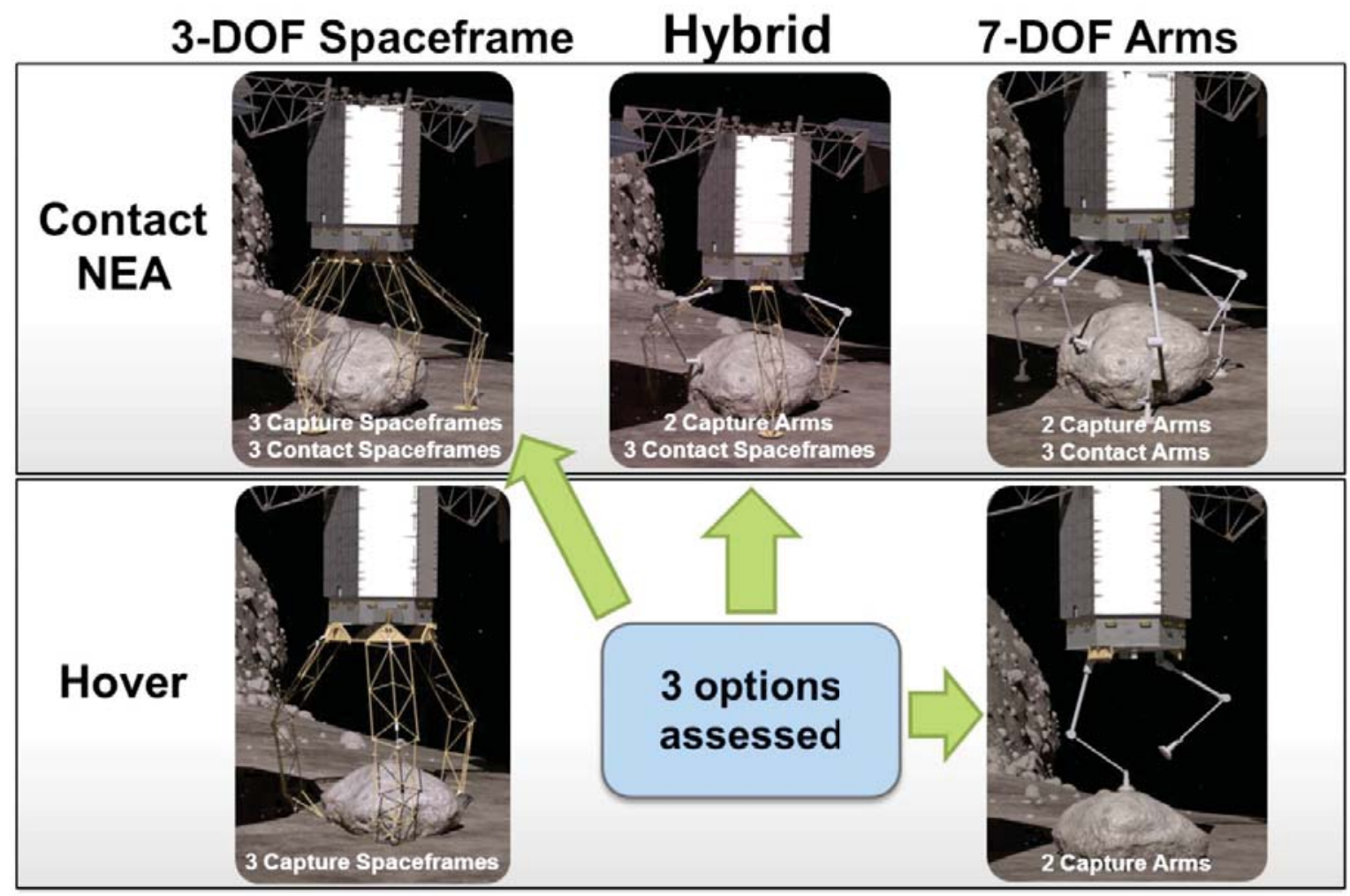

Figure 9: Proximity Operations and Capture Module Options (Image Credit: NASA/AMA, Inc.).

defense demonstration phases. The Wide Field of View (WFOV) and LIDAR are fixed to the nadir deck of the Capture Module to provide imagery of the boulder though descent and capture. Fig. 10 describes the sensor capabilities and architecture as it pertains to the Proximity Operations, which are discussed in Section VII.

The Robot Subsystem design consists of two 7-DOF arms currently under development at NASA's Satellite Servicing Capabilities Office (SSCO) for use on proposed satellite servicing missions. The arms take advantage of an extensive development program and include a new SSCO-designed Advanced Tool Drive System (ATDS). ${ }^{16}$ The ATDS includes tool change out capability, several tool drives, and two pointable cameras to provide imagery of the boulder and robotic workspace. The boulder capture end-effector tool is the Jet Propulsion Lab (JPL) Microspine Gripper, which uses hundreds of fishhook-like spines to opportunistically grab the surface features of natural objects. ${ }^{17}$ Fig. 11 shows the configurations of the 7-DOF Arm and Microspine Gripper (different scales). The robot subsystem also allows for boulder examination and sample preparation while in transit to cislunar space. After collecting the boulder and ascending from the surface, the arms position the boulder for restraint in the Contact and Restraint Subsystem (CRS), which is described below, and then can stow the Microspine tool and acquire other tools for boulder characterization and sample collection and caching. The crew's EVA time during the ARCM will be very limited and any site work done before the crew's arrival will relieve the crew of the need to perform lengthy coring and other sample gathering tasks.

The Contact and Restraint Subsystem (CRS) is required to arrest the descent of the spacecraft and stabilize it on the surface of the asteroid, as well as providing the means of departing the surface using a mechanical push-off. This approach has been investigated to avoid directly pluming the surface of the NEA with the RCS so as to minimize contaminating the spacecraft's solar arrays and other sensitive components with dust or larger particles. The CRS design shown in Fig. 12 consists of a set of three Spaceframe arms, each with three degrees of freedom and contact pads at their tips. The contact pads accommodate geological/geographical context sample collectors to accompany the captured boulder. 


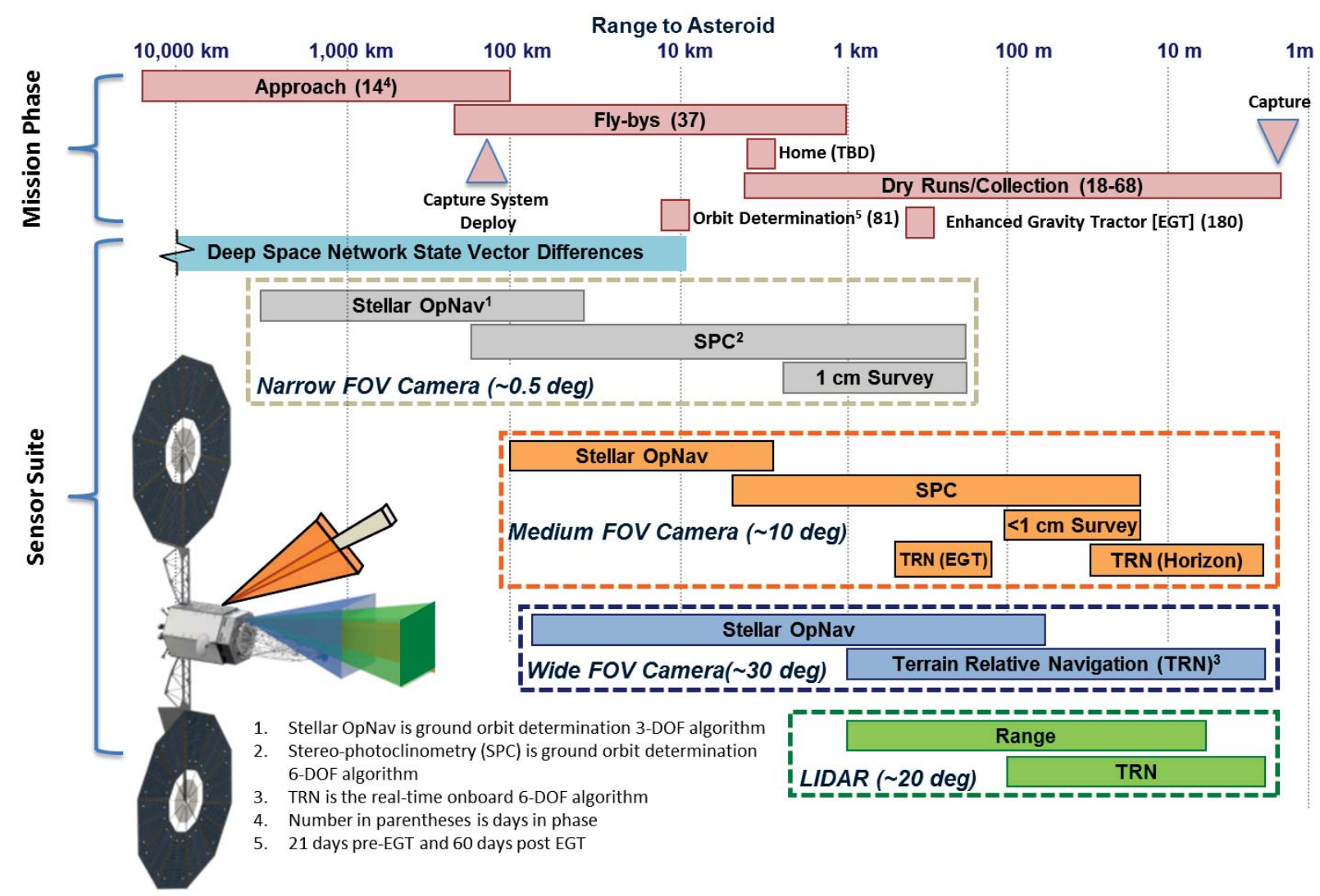

Figure 10: Option B Proximity Operations Sensor Suite and Operations.

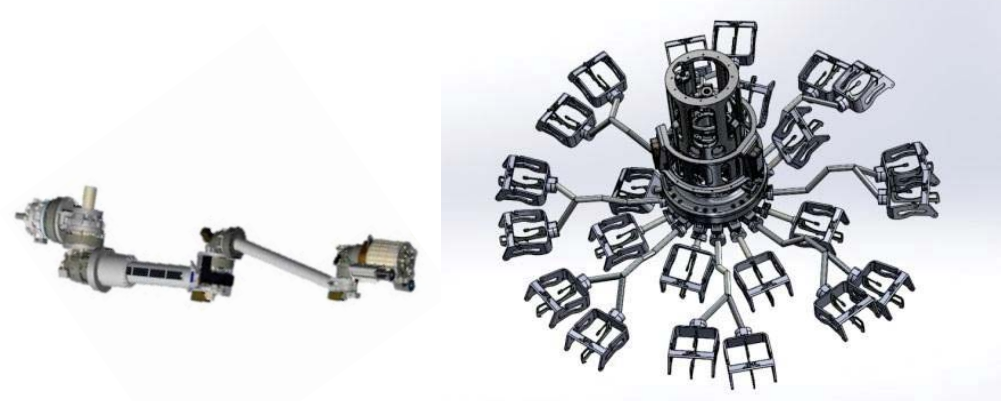

Figure 11: 7-DOF Arms and Microspine End Effector at Different Scales (Image Credits: NASA/JPL).

With the micro-gravity environment and the centrifugal force due to the asteroid's rotation it was also assumed that "landing" would be preferred for stability and operational simplicity with the RCS firing to provide additional force against the surface to further accommodate the reaction forces of capturing the boulder. To reduce the likelihood of pluming the surface with the RCS while descending, the contact arms are designed to absorb the momentum of the spacecraft using electrically driven linear actuators. Those same actuators are used to provide the push-off force needed to depart the surface with the captured boulder.

The CRS is shown in its extended position after launch in Fig. 12 and consists of three segments each built from aluminum tube stock of welded construction to form a no-wall open truss, or space frame. Segment rotation joints consist of spherical bearings providing rotational freedom with axial constraint while allowing for structural distortion from thermal expansion and contact with the irregular surface of the boulder. Flight heritage linear actuators are used to provide the high forces needed to drive the lengthy segments against the boulder and for the contact arms during descent and ascent. The boulder restraint requirement poses a challenge due to the wide variety 


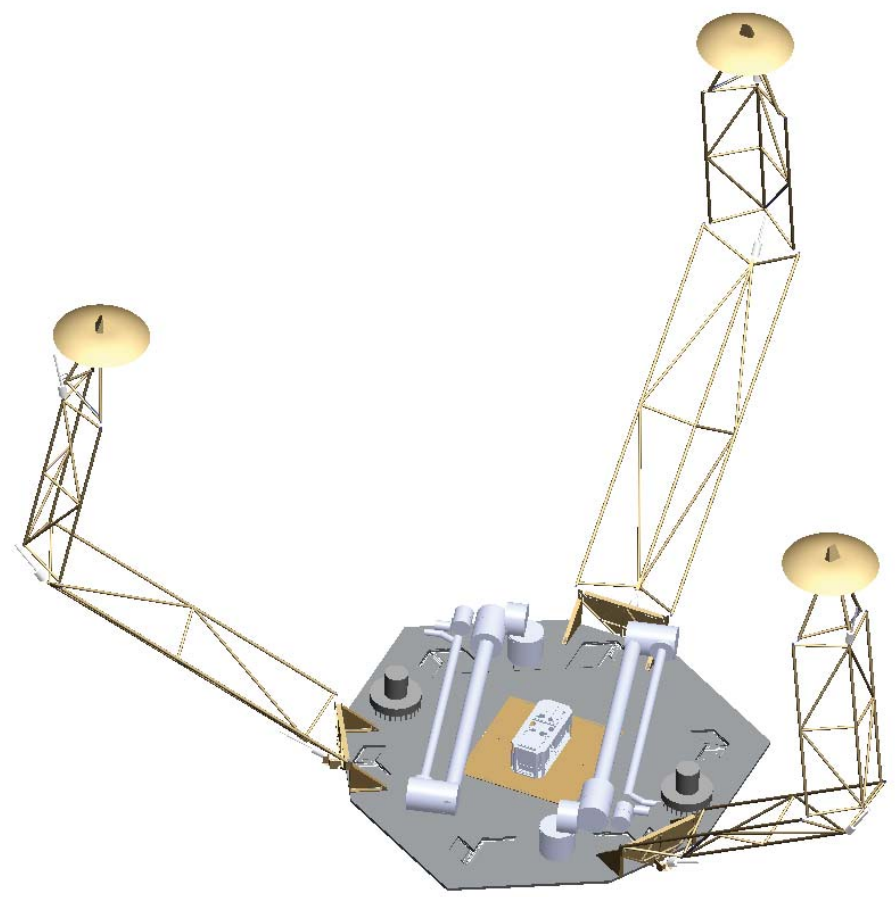

Figure 12: CRS in Extended Position (Post Launch).

of sizes and shapes of boulder it has to accommodate. The requirement of having to capture and restrain a spheroidal shape with various aspect ratios and ranging in major dimensions from 1 to 4 meters required numerous design iterations. The result is three identical arms with three different length segments and three different joint angle ranges all driven by three identical actuators. The design is a minimalistic method requiring no end effectors and is analogous to a large three finger end effector mounted to the nadir face of the spacecraft. The CRS also provides a ladder-like structure that can be used to assist in crew translation, stabilization and tool tethering while performing EVAs.

The Avionics Subsystem consists of three SpaceCube 2.0 data processors, one Vision Distribution and Storage Unit (VSDU), and one Remote Interface Unit (RIU). The SpaceCubes host all relative navigation and robotic control software in a 2-for-3 redundant configuration. The VSDU provides image storage and compression, and serves as a video router for all of the sensors, transmitting live, raw imagery to the SpaceCube and live or stored compressed video and other high speed telemetry to the Mission Module communications system for transmission to the ground. The RIU provides power switching services and miscellaneous other services to the payload.

The Mechanical Subsystem consists of the Capture Module structure, thermal hardware (heaters and payload avionics radiators), and all other miscellaneous hardware required to perform the Capture Module-allocated functions.

\section{Proximity Operations}

The proximity operations for Option B consist of four phases: 1.) Approach and Characterization; 2.) Dry-runs; 3.) Boulder Collection; and 4.) Planetary Defense Demonstration via the Enhanced Gravity Tractor (EGT) method. These operations are summarized here and are discussed in further detail in Ref. 18. For the Itokawa case study these operations require 400 days that include 50 days of asteroid and boulder characterization, 20 days of operations for the initial boulder collection attempt, 50 days of contingency operations in the event the initial boulder collection attempt is not successful, 260 days for demonstration and verification of EGT technique, and 20 days of margin. While the timeline for the main operations is shorter than the proximity operations for other robotic asteroid missions (e.g., OSIRIS-REx and Hayabusa), ARRM Option B relies heavily on the experience gained from previously flown missions and the extensive research and planning that has already been conducted during the preparation of future missions. Purposefully drawing from this knowledge minimizes mission unique operations and subsequently reduces mission risk. This was necessary in part because the operations timeline is also constrained by the competing desires to demonstrate a measureable deflection of an asteroid using a slow-push/pull technique,

13

American Institute of Aeronautics and Astronautics 
which takes significant time, and the desire to maximize the returned mass. As discussed earlier, any additional stay time at the asteroid for proximity operations reduces the return time of flight, which in turn reduces the boulder mass that the ARV EPS is capable of returning by the desired return date.

The Approach and Characterization Phase is depicted in Fig. 13 and begins at a range of around 1,000 km where the incoming trajectory will follow a straight line with a 45 degree Sun-Asteroid-ARV angle to $100 \mathrm{~km}$ and will last 14 days. During this time the lighting angle allows for good optical tracking by the NFOV cameras in order to refine the asteroid ephemeris and shape models. This phase also facilitates an update of the asteroid's attitude and spin states from previous knowledge. In the case of Itokawa, this prior knowledge is fairly precise and would require only minor updates. However, if the target is a previously unvisited asteroid with only ground-based information, detailed shape and spin models will need to be developed in real time.

After the global characteristics are determined, detailed mapping of the surface, potential boulders, and the gravity field is conducted through a series of fly-bys. The fly-bys each have a close approach distance of about $1 \mathrm{~km}$ and close approach velocities decreasing from $1.0 \mathrm{~m} / \mathrm{s}$ to $0.1 \mathrm{~m} / \mathrm{s}$. Targeting different areas of the asteroid and different lighting conditions, the NFOV and MFOV cameras on the 2-axis gimbal will map the entire surface to identify landmarks to be used by the TRN system while targeting close approach imaging on potential boulder collection sites. During these close approaches the cameras will provide centimeter-level resolution of the potential target boulders and the surrounding terrain. Seven days are allocated between each close approach to allow for downlink, ground processing, and uplink of targeting updates for the next fly-by.

Reducing the speed of the fly-bys allows more imaging time at higher resolution and also helps to refine the knowledge of the gravity field. Four fly-bys will provide adequate imaging time and gravity knowledge for Itokawa and other targets that have precursors, providing quality gravity models and imagery to identify potential target boulders. For previously un-visited NEAs, additional fly-bys will be needed to refine shape and spin models, develop detailed gravity models, and identify potential target boulders.

Following the fly-bys, the primary boulder target will be selected based on all the imagery and data collected. At this point the dry-run sequence will begin. In order to set a common point for each dry-run and descent trajectory to follow the same path a "Home Point" was designated at a range of $5 \mathrm{~km}$ and a 45 degree sun angle. Rather than hold position at this point, The ARV will simply pass through it at the beginning of each sequence. Once the command to begin the first dry-run is received, the ARV will maneuver to this point and then it will begin a passively-safe trajectory toward Waypoint 1 (WP1), which is defined to be outside of the bounding sphere of the asteroid and will be the point where, after passing through it, the ARV will begin targeting the surface. As shown in Fig. 14, an Intermediate Waypoint (IWP) was added for Itokawa due its highly non-spherical shape. This allows a passivelysafe trajectory from the Home Point to Waypoint 1 through this Intermediate Waypoint regardless of the spin state of Itokawa. This conservative operation is not needed as asteroids become more spherical.

Once the ARV arrives at Waypoint 1, the first dry-run is complete and the ARV completes a "Get Out Of Dodge" (GOOD) Maneuver that is a scripted, previously loaded maneuver to send the ARV on a slow drift trajectory away from the asteroid. Over the next seven days, the ARV will downlink telemetry data and imagery that will provide sub-centimeter resolution of the boulder and surrounding terrain, as well as updates to the boulder-local gravity field.

The ARV will then proceed back to the Home Point (HP) where it will begin the second dry-run by following the same path to Waypoint 1. From here the ARV will proceed to Waypoint 2 (WP2), which is at an altitude of $50 \mathrm{~m}$

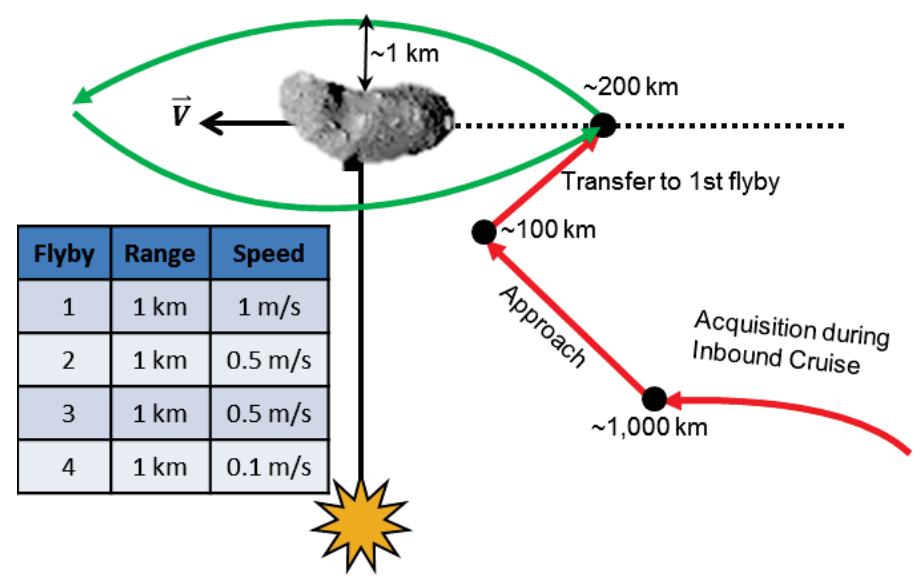

Figure 13: Approach and Characterization Phase. 


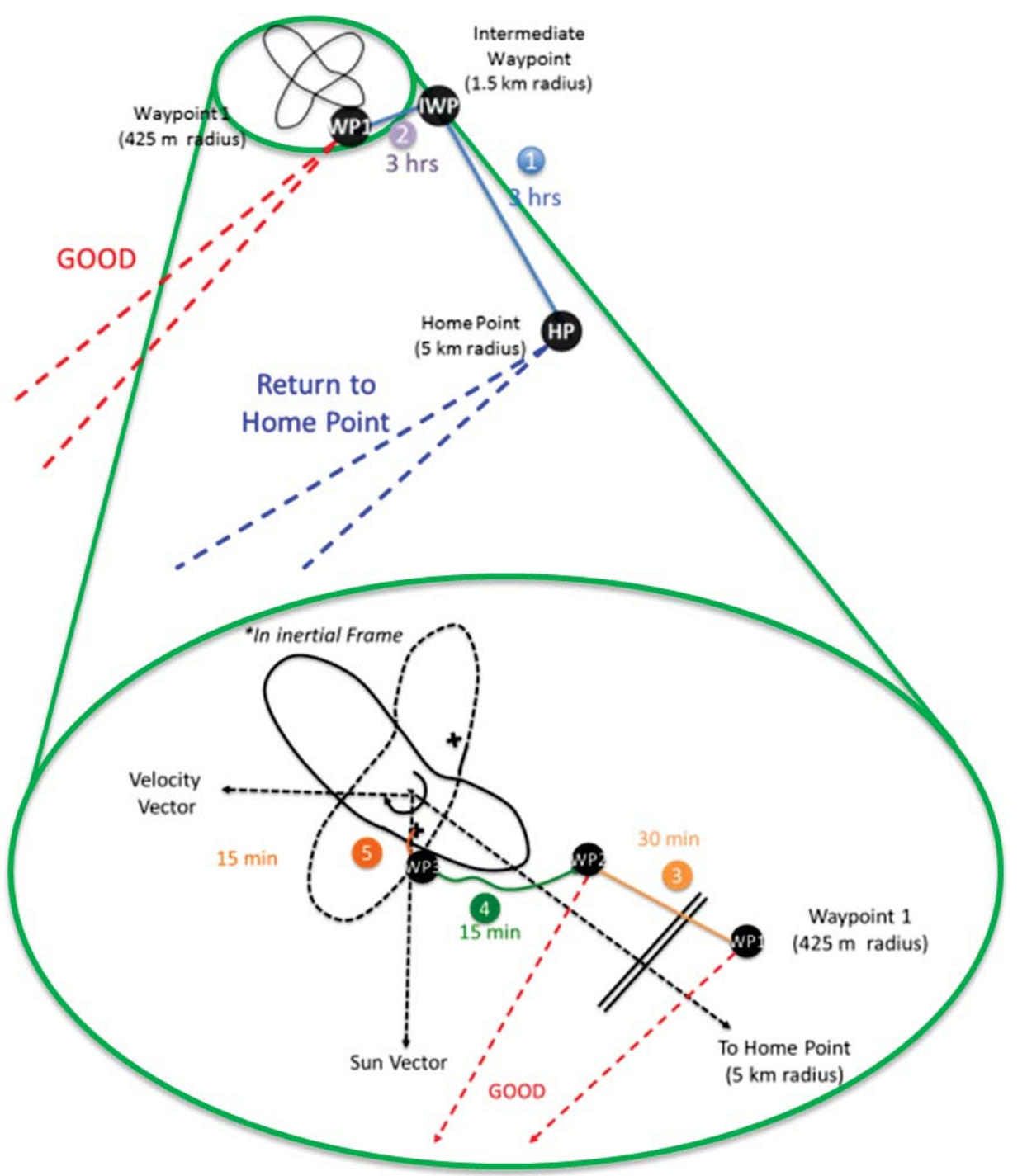

Figure 14: Dry-Run and Descent Trajectories.

directly above the target boulder. In order to validate the closed-loop TRN and control algorithms, the ARV matches the asteroid's spin rate and performs a short hold at Waypoint 2 while maintaining position directly above the boulder. This hold also allows the collection of additional high-resolution images under lighting conditions that will be seen during final descent and local gravity models to be validated. After the hold, another GOOD maneuver is executed, the data is downloaded and the ARV returns to the Home Point for the beginning of the boulder collection operations.

The boulder collection sequence begins by following the same path from the Home Point to Waypoint 2, at which point the ARV begins a vertical descent maintaining position directly above the boulder to Waypoint 3 (20 m altitude). This descent, as well as the descent from Waypoint 3 (WP3) to the surface, will rely solely on closed-loop TRN from the nadir-pointing WFOV camera and LIDAR tracking the boulder, as well as the side-looking NFOV and MFOV cameras that will be tracking other surface landmarks. At Waypoint 3 the vertical velocity may be nulled and from this point down to the surface there will nominally be no thrusting toward to the asteroid to prevent pluming contamination and debris creation. For a slow spinning asteroid such as Itokawa, the gravity is enough to overcome the centripetal acceleration and slowly pull the ARV towards the surface. For faster rotators, the ARV may need to use RCS jets to overcome the centripetal acceleration in order to descend to the surface while maintaining position directly above the boulder.

Upon contact, the CRS will attenuate the residual velocity and bring the ARV to rest on the surface. For Itokawa, this residual velocity was less than $5 \mathrm{~cm} / \mathrm{s}$ for a collection site on the Muses Sea. For faster spinners which require 
thrusting to descend, the contact velocity can be tailored even lower. While on the surface and throughout the collection operations, the RCS thrusters will provide a net $\sim 1 \mathrm{~N}$ of thrust above what is required to maintain contact. This will provide additional stability and keep the thrusters warm should additional thrust be needed due to forces experienced during boulder collection.

After the boulder is securely captured, the CRS will be used to push off the surface to break the cohesion between the boulder and the surface and to impart an initial velocity to the combined ARV and boulder system. Nominally this velocity will be slightly above escape velocity to put the ARV on a slow drift trajectory away from the asteroid. If escape velocity is not met, RCS thrusters will be used to add the required impulse. During the first 50 meters of ascent, the ARV will use the RCS sparingly only to null any tip rates in order to ensure the solar arrays will not contact the surface. Past this point the RCS will remove the residual motion to achieve a sun-pointing attitude.

At this point the boulder has been captured, full solar power has been restored, a high-bandwidth communications link with Earth is established for downlink, and the ARV is on a passively-safe trajectory away from the asteroid. Prior to any significant maneuvers, a series of small burns will be executed in order to refine the mass properties of the combined system. For the hybrid capture system, the CRS then performs the restraint sequence as the ARV with updated mass properties maneuvers to complete the ascent phase.

With the boulder restrained, the ARV begins a three-week orbit determination period to refine the postinteraction asteroid orbit prior to the planetary defense demonstration. The baseline planetary defense technique for Option B is the Enhanced Gravity Tractor (EGT) technique. As shown in Fig. 15, the EGT technique investigated uses a halo orbit around the asteroid's orbital velocity vector $(\vec{v})$ with the SEP thrusters throttling to match the gravitational force and maintain a constant separation. The gravitational attraction along the radial vector $(\vec{r})$ results in a net force along the velocity vector. The halo orbit is designed with a radius (y) to maintain a safe minimum distance from the NEA and eliminate pluming of the surface (325 m for Itokawa operations). The halo orbit eliminates the need for large thruster gimbal angles that would be required if the ARV maintained position along the velocity vector to avoid pluming on the NEA and negating the gravitational attraction between the ARV and the NEA. For Itokawa with a nominal $7 \mathrm{t}$ boulder, 90 days of tractoring is required in order to achieve a measurable deflection with 3- $\sigma$ certainty. The nominal timeline and propellant budget includes 180 days of tractoring to provide margin for any contingencies periods where the ARV would enter into a safe mode and temporarily halt tractoring activities. The estimated tractoring period required to achieve a measurable deflection if no boulder is collected is achievable by increasing the tractoring time to 180 days.

Following the tractoring activities, the ARV will maneuver to a safe distance and wait for the achieved deflection to propagate and for proper orbital alignment with Earth for verification of the deflection. The verification will be achieved through direct range measurements of the ARV combined with the relative range measurements between the ARV and the asteroid.

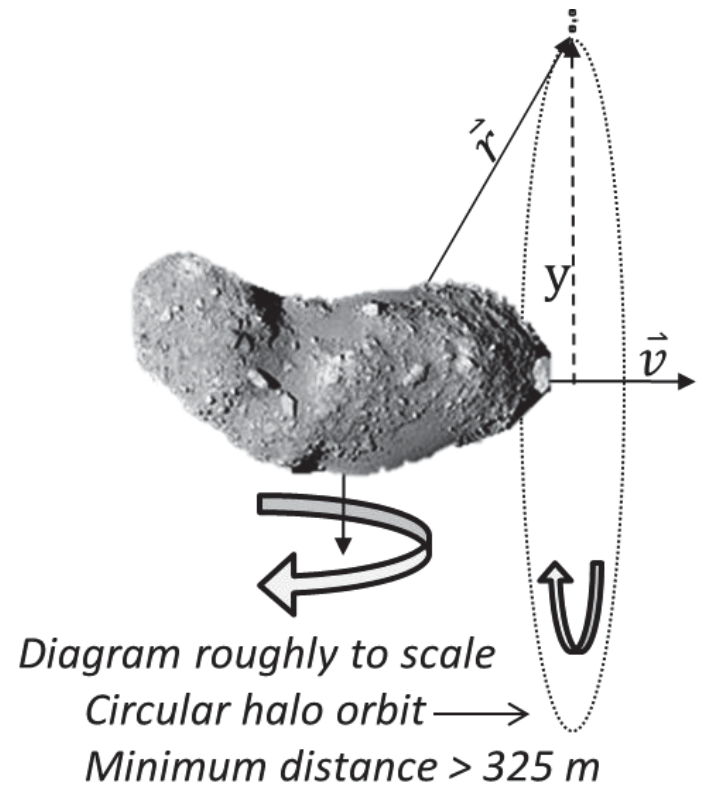

Figure 15: EGT Demonstration Orbital Geometry (Itokawa Image Credit: JAXA). 


\section{Planetary Defense Demonstration Options}

One of the key findings of the 2010 National Research Council Committee report titled "Defending Planet Earth: Near-Earth Object Surveys and Hazard Mitigation Strategies" was that "no single approach to mitigation is appropriate and adequate for completely preventing the effects of the full range of potential impactors, although civil defense is an appropriate component of mitigation in all cases. With adequate warning, a suite of four types of mitigation is adequate to mitigate the threat from nearly all NEOs except the most energetic ones." ${ }^{" 19}$ Besides civil defense as a mitigation technique, the three planetary defense techniques highlighted in the report were gravity tractor as a slow push/pull technique, along with kinetic impactor and nuclear detonation as rapid "impulsive" techniques.

As mentioned above, the primary planetary defense demonstration proposed for ARRM Option B is the Enhanced Gravity Tractor (EGT), which uses the collected boulder to augment the mass of the ARV. Additional approaches, such as a Kinetic Impactor (KI) or Ion Beam Deflection (IBD) can also be performed during the mission. The KI demonstration requires the addition of a secondary impacting spacecraft to be launched with the ARV or separately, along with the coordination of the impactor to strike the NEA near the end of the NEA visit. A KI demonstration in this manner is not applicable to Option A. The IBD approach utilizes the ion plasma from the SEP thrusters to impinge against the NEA's surface and impart a force on the target. The effectiveness of the EGT and IBD approaches for a given size NEA primarily depends on the mass of the collected boulder and the SEP thruster plume divergence, respectively. Additionally, the collection of engineering data during surface interaction and the potential for testing some of the hardware and operations that would be applicable to a nuclear detonation is also possible for Option B.

There are a number of reasons for selecting EGT for demonstration on Option B of the ARRM. First, the approach minimizes additional mission costs and risks to demonstrate a viable PD technique by leveraging the collected boulder mass, which is nominally being acquired. Second, it is relevant to hazardous-size NEAs with an efficiency that increases as the boulder and NEA masses increase. For an NEA the size of Itokawa, EGT requires less time than IBD to impart a specified $\Delta \mathrm{V}$ on the target if a sufficiently massive boulder is captured, and uses approximately half the propellant required for IBD ( 300 kg vs. $\sim 600 \mathrm{~kg})$ since the IBD approach requires propulsive capability to compensate for the reaction force of the plasma beam directed at the NEA. Fig. 16 provides a comparison of the EGT with $10 \mathrm{t}$ and $20 \mathrm{t}$ boulders and the IBD to impart a $0.1 \mathrm{~mm} / \mathrm{s} \Delta \mathrm{V}$ on a range of NEAs sizes. Finally, the EGT technique allows the ARV to maintain safe, constant distance from NEA after retrieval while demonstrating extended proximity operations near the asteroid.

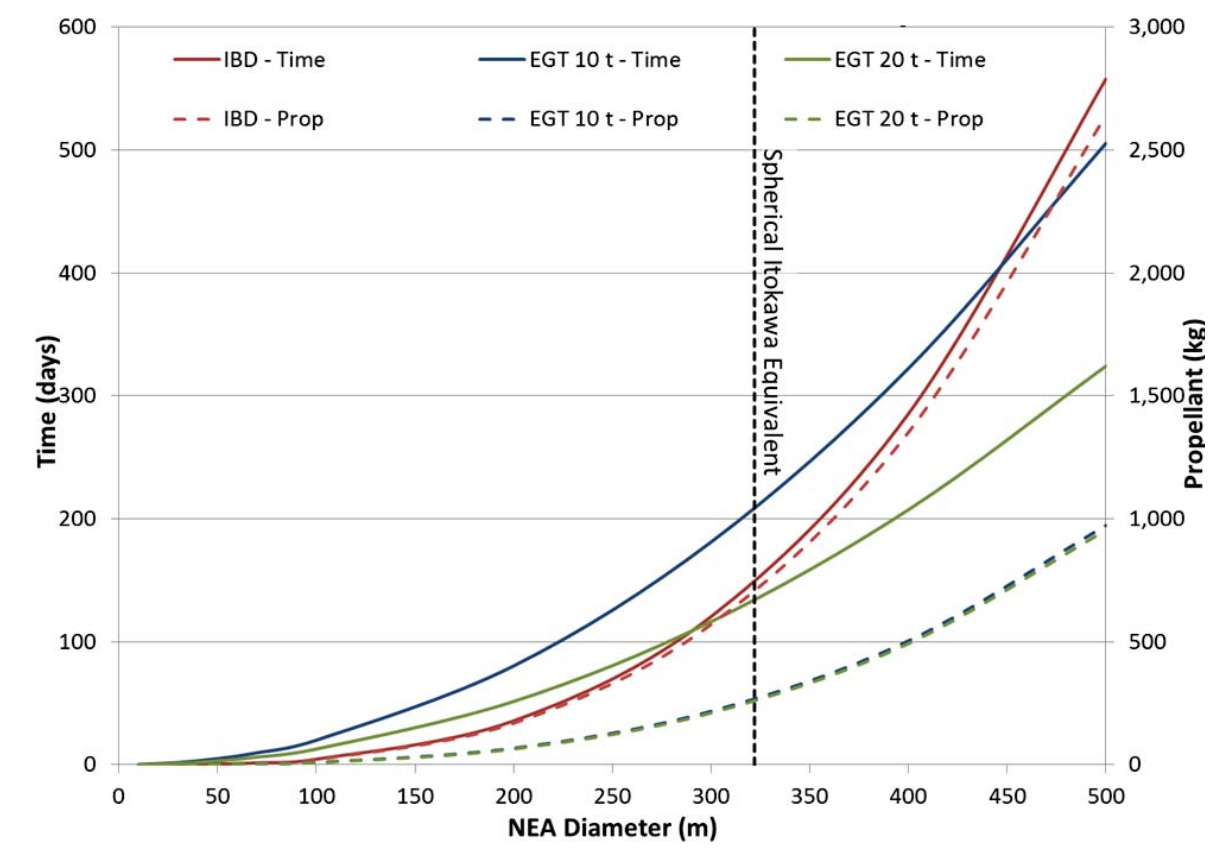

Figure 16. Enhanced Gravity Tractor Compared to Ion Beam Deflection to Impart $\Delta V=0.1 \mathrm{~mm} / \mathrm{s}$.

17

American Institute of Aeronautics and Astronautics 
The use of the mass collected in-situ and the ability of multiple ARVs to orbit in formation to provide the necessary velocity change greatly increases the applicability of the EGT technique, which allows, with sufficient warning time the EGT technique to be used to divert hazardous NEAs. For an actual deflection mission to avoid a future Earth impact, the collected NEA material, which could be a single boulder, multiple boulders, or regolith, would not need to be returned to Earth. Therefore, significantly more mass could be collected to provide mass augmentation than cannot be achieved during the ARRM. With the current thrust levels available from the EPS, imparting a $\Delta \mathrm{V}$ of $0.5 \mathrm{~cm} / \mathrm{s}$ on an Itokawa-size NEA ( 320 m equivalent diameter) with a single ARV could be accomplished with the collection of $\sim 275 \mathrm{t}$ of asteroidal material and would require $\sim 3.4$ years of tractoring and $\sim 6 \mathrm{t}$ of xenon propellant. However, two ARVs working in tandem would only require $\sim 1.7$ years and $\sim 3 \mathrm{t}$ of propellant each. Without the collected mass, a single ARV would need 120 years and $\sim 15 \mathrm{t}$ of xenon propellant to impart the same velocity change. It should be noted that these estimates are based on a fixed ARV mass. In actuality the ARV mass would change as the propellant load is consumed, but it would still be dominated by the mass of the collected material.

\section{Programmatic Robustness and Development}

The ARRM Option B mission and flight system have been designed to allow the mission to be robust programmatically. The robust target set and scalability of the capture system allows the mission to be robust to launch date, providing NASA with flexibility in when and how to execute the mission. The functions have been distributed within the modular flight system and Capture Module in ways that allow the schedule risk to be minimized while also accommodating the use of different providers for each module. The Capture Module cost and schedule reserve allocations are also appropriately sized for a mission in formulation, with schedule reserves complying with established design principles and cost reserves margin in excess of $34 \%$ for all three Capture Module concepts.

The NEA target set available to the mission allows a multi-ton return mass for a wide range of departure and return dates, as shown in Table 1 in Section IV. This rich target set availability provides NASA with flexibility in terms of when the mission is provided with authority to proceed and the planned development duration, allowing the mission to more easily accommodate potential budget profiles and reducing the impact of potential budget uncertainties. In addition, once design and development has started, the availability of multiple targets provides mission management options in the event of new information attained from planned precursors or unforeseen issues. Once launched, boulder target selection and return mass can be also adjusted, providing the mission with the flexibility to increase the value in response to internal or external factors. For example, in the event of changes to the Agency's planned crew launch cadence after an ARMM Option B launch, the mission could pursue the capture of larger boulders by slipping the planned return date.

The design, build, integration, and test schedule for the ARRM Option B Capture Module has been structured to allow an integrated test of the Capture Module to occur prior to system-level Integration and Test (I\&T), and minimize the impact of many of the module subsystems on the module critical path. The desire for an integrated test of the Capture Module led to the modular structure described in Section III, with the Capture Module functionality including all critical capture systems. For the Hybrid and Spaceframe options, the Spaceframe capture/contact arms design, development, integration, and test activities are the Capture Module's critical path. To minimize the duration of that path, the module I\&T plan, shown in Fig. 17, has been structured so that the other module subsystems will be integrated and tested prior to the integration of the Spaceframe arms for final module I\&T.

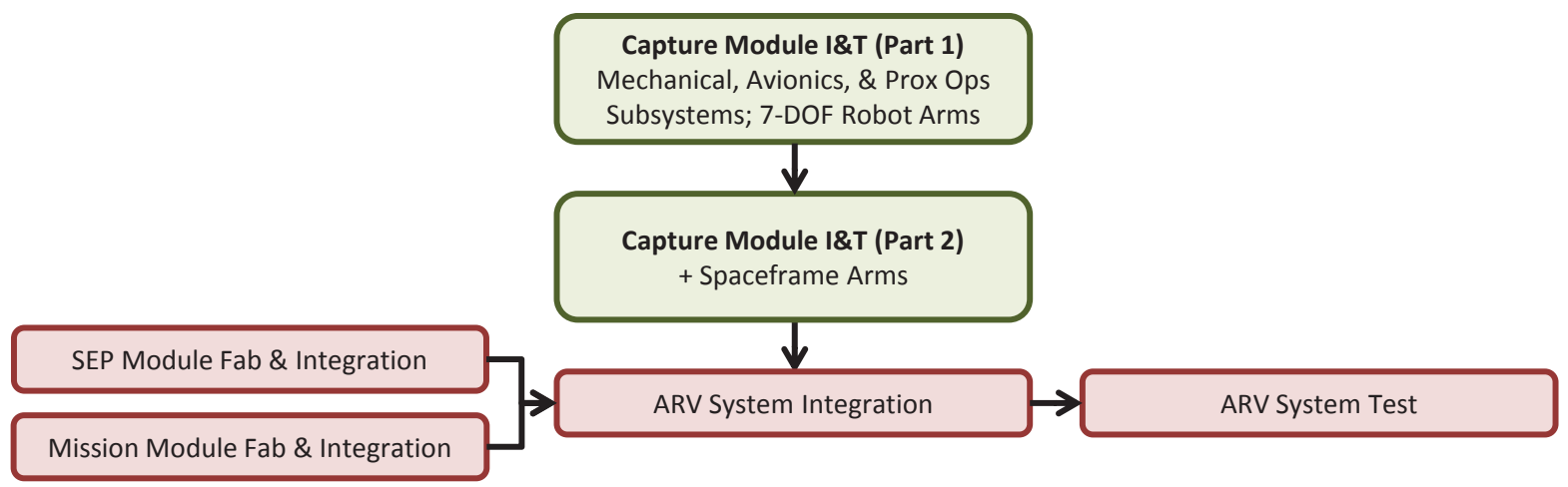

Figure 17. ARRM Option B I\&T Approach for Hybrid Capture Module. 


\section{ARRM Extensibility}

This Asteroid Redirect Mission includes aspects of extensibility related to both the robotic and crewed segments. This paper summarizes the extensibility of the ARRM. Please refer to Ref. 20 for a detailed discussion of ARCM and ARRM extensibility. For the ARRM, extensibility falls into three general categories for both future human and robotic missions. The first is Extended Missions where the ARV, as designed, is used for post-ARCM human and robotics missions by NASA, commercial, academic and/or international partners. Included in this first category would also be an "end-of-life mission" where the ARV by itself or along with any remaining asteroidal material would perform a final mission of interest near the end of its operational lifetime. An example of this would be the use of the ARV and leftover asteroidal mass to demonstrate the kinetic impactor technique on an asteroid or comet. The second category is Augmented Missions where the ARV is used in conjunction with additional vehicles/modules to enhance the available capabilities. An example of this would be the addition of a pressurized module to provide additional habitable volume for crewed visits in cislunar space. A second example of an augmented mission would be to utilize a modified version of the Capture Module with existing SEP and Mission Modules to return a boulder from one of the Martian moons as depicted in Fig. 18. This second example assumes that sufficient propellant is available to conduct this mission. Having the option to refuel the ARV (RCS and SEP) would be extremely valuable and provide the ability maximize the benefits and use of the ARV for both of these categories. The final category is Evolved Missions where advanced versions of the ARV modules and subsystems, including higher power SEP would be used to perform future robotic or human missions which require significantly increased capabilities. A robotic mission to the Martian moons could also be included in this category as a follow-on mission to ARRM using an evolved version of the ARV (e.g., additional power and xenon propellant). A second example of this would be the use of a higher power SEP Module and Capture Module subsystems to deliver a habitat to surface of Phobos to support a human mission to the Mars system as depicted in Fig. 19.

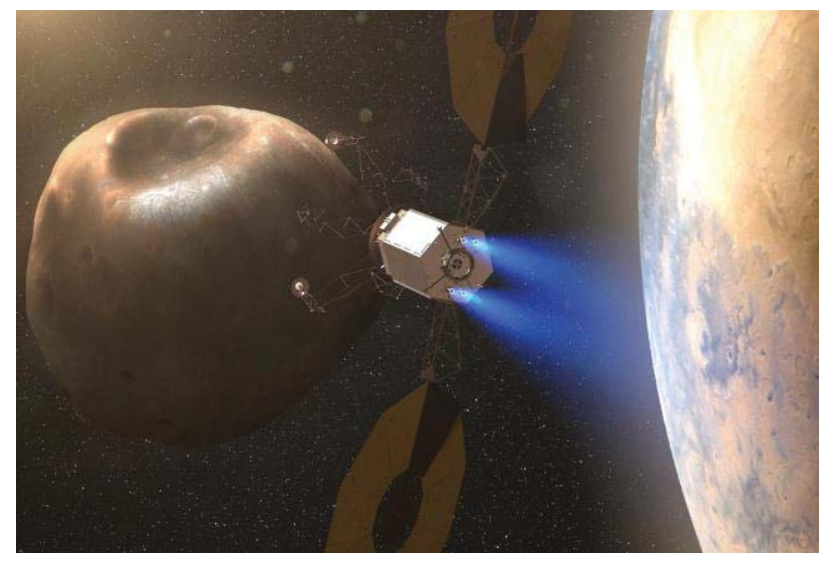

Figure 18. Extensibility of ARV to Boulder Retrieval at Phobos (Image Credit: NASA/AMA, Inc.).

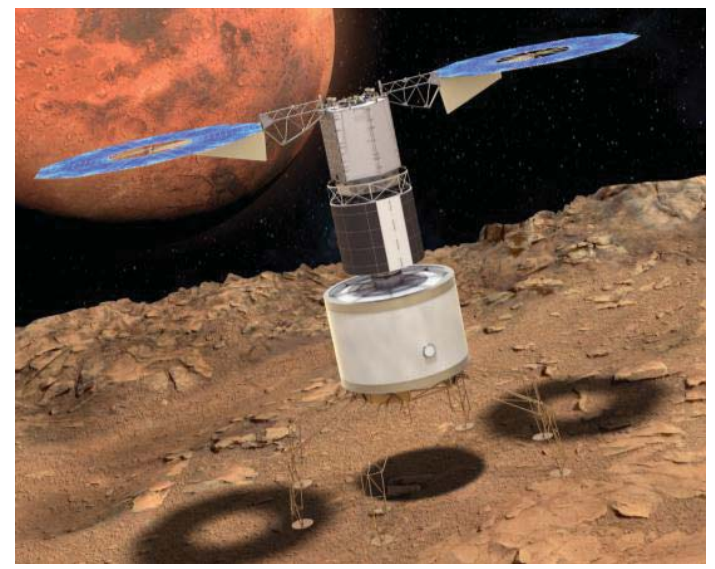

Figure 19. Concept for Evolved ARV Integrated with a Phobos Surface Habitat (Image Credit: NASA/AMA. Inc.).

19

American Institute of Aeronautics and Astronautics 


\section{A. Solar Electric Propulsion}

The ARV, with $50 \mathrm{~kW}$ solar arrays and a $40-\mathrm{kW}$ electric propulsion system with the capacity to hold approximately 10 metric tons of usable xenon propellant, is a spacecraft that is very capable for use in future human and robotic exploration missions. These missions are wide-ranging and include supporting long-duration human missions in cislunar space to interplanetary robotic exploration of the solar system. The ARV modules and subsystems, through modularity and scalability, can be evolved to facilitate more demanding missions such as cargo delivery and human missions to Mars. Human Mars missions will require higher power solar arrays and electric propulsion systems. The current ARV and/or its subsystems could be used in a modular manner or upgraded as the technologies advance. The solar array systems being developed are planned to be extensible to $125 \mathrm{~kW}$ per wing. This increased power can either be combined with a greater number of EPS strings at the current power and thrust levels or with more capable electric propulsion systems. Current solar array and electric propulsion systems technology maturation efforts by STMD for the ARRM will advance these critical technologies to enable moredemanding NASA's missions in the future and will advance high-efficiency in-space propulsion that can be incorporated into commercial spacecraft.

\section{B. Capture Module}

The use of Capture Module's subsystems to interact with an asteroid and capture a boulder from its surface has many applications to future NASA missions as well as commercial companies and international partners. The extensible activities include satellite servicing and refueling, providing surface contact and manipulation for a future crewed mission to NEAs or the Martian moons, and providing critical experience operating around and on a lowgravity planetary body. The use of planned routines with fault tolerance for asteroid surface contact and boulder collection operations will be required for future autonomous operations in deep space. In addition, the Microspine technology can be used for future robotic exploration of lava tubes on the Moon and Mars, Martian cliffs, and lowgravity bodies like NEAs, Phobos, Deimos, and Ceres. The hybrid approach optimizes functionality and maximizes extensibility of the concept. Fig. 20 provides a depiction of how the Capture Module subsystems could be utilized as part the Multi-Mission Space Exploration Vehicle (MMSEV) on the surface of Phobos.

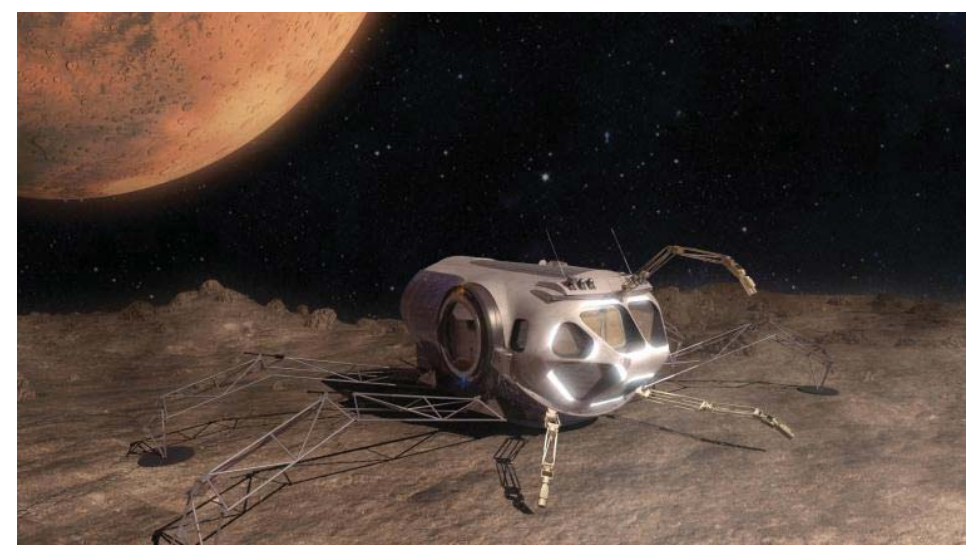

Figure 20. MMSEV with ARV Capture Module Subsystems on the Surface of Phobos (Image Credit: NASA/AMA, Inc.).

\section{Asteroid Resource Utilization}

Water is a critical resource for future human space exploration and can be used for a variety of purposes, including propellants, radiation shielding, thermal control, human consumption, as well as a variety of non-potable applications (cleaning, plant growth, etc.). Carbonaceous asteroids may contain up to $20 \%$ water by mass in the form of hydrated minerals and a similar mass percentage of other extractable volatiles (e.g., carbon dioxide, nitrogen, and carbon monoxide). Carbonaceous asteroids also have low compressive strength. This property simplifies cutting, crushing, and processing, which could be a key target attribute for resource extraction and recovery. Oxygen from mineral oxides, metals (e.g., iron, nickel, and platinum group metals), and bulk materials that can be used for radiation shielding and the construction of various structures are also plentiful in NEAs.

Determining the composition of the target NEA is critical and targeting a large, well-characterized carbonaceous NEA in Option B significantly increases the potential for returning water/volatile-rich material. Returning the right type of asteroidal material to cislunar space would allow ISRU demonstrations to be conducted to determine how 
successfully the bulk asteroidal material may be converted into useful products. This would be enabling for future human exploration of the solar system and could be critical in advancing commercial efforts to mine asteroids. In addition to resource extraction, the analysis and understanding of water and water-bearing minerals from asteroids is extremely important to our understanding of solar system formation and the origins of life on Earth.

\section{Planetary Defense}

Although slow push/pull planetary defense techniques, such as gravity tractor and ion beam deflection, may be demonstrated as part of both ARRM options, the relevance and extensibility of the techniques are significantly improved by conducting a mission to a hazardous-size NEA. The size of NEA targeted by the Option A is below the threshold that can typically survive entry through the Earth's atmosphere, and therefore is not of a hazardous-size. Option B provides critical planetary defense experience by operating at a large NEA, both through sustained orbital operations and extended surface interactions. It also allows the demonstration of the Enhanced Gravity Tractor (EGT) that can be used to significantly reduce the operational time needed for this approach to be effective against an actual impactor. Finally, the option to demonstrate a kinetic impact technique, which is the most likely option for an impactor with limited warning time, is only available with Option B.

The additional extensible approach for planetary defense that can be derived from the ARRM is the use of the ARV and the captured boulder to act as a kinetic impactor against an asteroid or comet threat. This option needs further investigation to determine the extent of its applicability, but with sufficient xenon propellant reserves, or the ability to refuel the ARV, having this asset in the Earth-Moon system could provide a basic "on call" planetary defense capability. The reality is that that no dedicated planetary defense system exists and funding one is unlikely due to the infrequency of Earth impacts. Developing the technologies, systems, and operational approaches for efficiently moving large amounts of useful asteroidal material and for facilitating its processing and utilization also helps us to be prepared to divert a future impactor. The ARRM provides a critical first step to an integrated solution to planetary defense by leveraging the resources of NEAs and their economic potential.

\section{Summary and Forward Work}

The Asteroid Redirect Robotic Mission is a critical component of NASA's Asteroid Initiative. The mission option of robotically capturing a multi-ton boulder from the surface of a larger NEA and returning it to cislunar space best addresses a broad set of stakeholder interests, including human exploration, science, planetary defense, and commercial and international partnerships. Option B can leverage precursor missions and existing Agency capabilities to provide an attractive set of candidate NEAs that are robust to changes in launch and return dates. The option provides centimeter-level characterization over an entire large NEA and sub-centimeter level characterization of multiple candidate boulders prior to selection and capture. Option B also provides the opportunity for hosted payloads from commercial, academic, and international partners. This ARRM option provides critical experience operating at a low-gravity planetary body, including extended surface interactions and the ability to demonstrate multiple deflection strategies on a hazardous-size NEA, including the option to test a kinetic impact approach. Finally, targeting a large, well-characterized NEA significantly increases the potential for returning water/volatilerich material from a carbonaceous NEA, which could be instrumental in advancing the fledgling asteroid mining industry.

Work on the ARRM Option B approach is continuing through a series of risk reduction tasks combined with additional mission design and analyses. A multi-center NASA team is currently focused on the design of the hybrid option, refining the proximity operations, performing expanded mission design for candidate NEAs, and testing key aspects of the approach. These activities are being conducted in preparation for a mission downselect between Options A and B planned in December of 2014 and a Mission Concept Review in February of 2015.

\section{Acknowledgments}

The authors would like to thank the entire cross-Agency team for the tremendous amount of work that has been performed on the Robotic Boulder Capture Option for ARRM. The Option B teams includes civil servants and contractors from Langley Research Center, Goddard Space Flight Center, Johnson Space Center, Glenn Research Center, Kennedy Space Center, Marshall Space Flight Center, Ames Research Center, NASA Headquarters, Jet Propulsion Laboratory (JPL), Colorado Center for Astrodynamics Research - University of Colorado at Boulder, and the University of Alaska Fairbanks. Additionally, the authors would like to thank the Analytical Mechanics Associates, Inc. (AMA, Inc.) personnel in the Advanced Concepts Laboratory at NASA LaRC, Dave Helton, Josh Sams, Bob Evangelista, Christopher Keblitis, and Kevin Greer, for the outstanding computer-generated graphics included in this paper. 


\section{References}

${ }^{1}$ Brophy, J. R., et al., “Asteroid Retrieval Feasibility Study,” Keck Institute for Space Studies Report, April 2012.

${ }^{2}$ Strange, N., et al., "Overview of Mission Design for NASA Asteroid Redirect Robotic Mission Concept," $33^{\text {rd }}$ International Electric Propulsion Conference, The George Washington University, Washington, D.C., October 2013.

${ }^{3}$ Mazanek, D. D., Brophy, J. R., and Merrill, R. G., “Asteroid Retrieval Mission Concept - Trailblazing Our Future in Space and Helping to Protect Us from Earth Impactors," $3^{\text {rd }}$ IAA Planetary Defense Conference, Flagstaff, AZ April 2013.

${ }^{4}$ Merrill, R. G., Qu, M., Vavrina, M., Englander, J., and Jones, C., "Interplanetary Trajectory Design for the Asteroid Robotic Redirect Mission Alternative Approach Trade Study,” AIAA/AAS Astrodynamics Specialist Conference, August 2014.

${ }^{5}$ Landau, D., McElrath, T., Grebow, D., and Strange, N., "Efficient Lunar Gravity Assists for Solar Electric Propulsion Missions," Paper AAS 12-165, Feb. 2012.

${ }^{6}$ McElrath, T., et al., "Using Gravity Assists in the Earth-Moon System as a Gateway to the Solar System," Paper GLEX 2012.05.5.2x12358, May 2012.

${ }^{7}$ Keil, K., “Thermal Alteration of Asteroids: Evidence from Meteorites,” Planetary and Space Science, Vol. 48, Issue 10, p. 887-903, 2000.

${ }^{8}$ Taylor, P. A., Howell, E. S., Nolan, M. C., Thane, A. A. "The Shape and Spin Distribution of Near-Earth Asteroids Observed with the Arecibo Radar System," Abstract 302.07, $44^{\text {th }}$ Division for Planetary Sciences Meeting, Reno, NV, October 2012.

${ }^{9}$ Fujiwara, A., et al., “The Rubble-Pile Asteroid Itokawa as Observed by Hayabusa,” Science, Vol. 312, p. 1330-1334, 2006.

${ }^{10}$ Miyamoto, H., et al., "Regolith Migration and Sorting on Asteroid Itokawa," Science, Vol. 316, p. 1011-1014, 2007.

${ }^{11}$ Noviello, J. L., Barnouin, O. S., Ernst, C. M., and Daly, M., "Block Distribution on Itokawa: Implications for Asteroid Surface Evolution," Abstract 1587, 45 ${ }^{\text {th }}$ Lunar and Planetary Science Conference, Woodlands, TX, March 2014.

${ }^{12}$ Nolan, M. C., et al., "Shape Model and Surface Properties of the OSIRIS REx target Asteroid (101955) Bennu from Radar and Lightcurve Observations,” Icarus, Vol. 226, p. 629-640, 2013. 2011.

${ }^{13}$ Busch, M. W., et al., "Radar Observations and the Shape of Near-Earth Asteroid 2008 EV5,” Icarus, Vol. 212, p. 649-660,

${ }^{14}$ Yurimoto, H., et al., "Oxygen Isotopic Compositions of Asteroidal Materials Returned from Itokawa by the Hayabusa Mission,” Science, Vol. 333, p. 1116-1119, 2011.

${ }^{15}$ Belbin, S. P., and Merrill, R. G., "Boulder Capture System Design Options for the Asteroid Robotic Redirect Mission Alternate Approach Trade Study," AIAA SPACE 2014, August 2014.

${ }^{16}$ Roberts, B., and Pellegrino, J., "Robotic Servicing Technology Development,” AIAA 2013-5339, AIAA Space 2013, San Diego, CA, 10-12 September 2013.

${ }^{17}$ Parness, A., et al., "Gravity-independent Rock-climbing Robot and a Sample Acquisition Tool with Microspine Grippers," Journal of Field Robotics 30, No. 6, p. 897-915, 2013.

${ }^{18}$ Reeves, D.M., Naasz, B.J., Wright, C.A., Pini, A.J., "Proximity Operations for the Robotic Boulder Capture Option for the Asteroid Redirect Mission,” AIAA SPACE 2014, August 2014.

${ }^{19} 2010$ National Research Council Committee report titled "Defending Planet Earth: Near-Earth Object Surveys and Hazard Mitigation Strategies,” The National Academies Press, Washington, D.C., 2010.

${ }^{20}$ McDonald, M. A., et al., "Extensibility of Human Asteroid Mission to Mars and Other Destinations," AIAA SpaceOps 2014 13th International Conference on Space Operations, May 2014. 